\title{
Medida de Gabriel-Roiter e o Teorema de Roiter
}

Eliza Hidemi Sadaike Miyazaki

\author{
DISSERTAÇÃO APRESENTADA \\ $\mathrm{AO}$ \\ INSTITUTO DE MATEMÁTICA E ESTATÍSTICA \\ DA \\ UNIVERSIDADE DE SÃO PAULO \\ PARA \\ OBTENÇÃO DO GRAU DE MESTRE \\ EM \\ MATEMÁTICA \\ Área de Concentração: Álgebra \\ Orientador: Prof. Dr. Flávio Ulhoa Coelho
}

-São Paulo, 20 de maio de 2005 
Este exemplar corresponde à redação

final da dissertação devidamente corrigida e defendida por

Eliza Hidemi Sadaike Miyazaki

e aprovada pela comissão julgadora.

São Paulo, 20 de maio de 2.005.

Banca examinadora:

- Prof. Dr. Flávio Ulhoa Coelho (orientador) - IME-USP

- Prof. Dra. Maria Izabel Ramalho Martins - IME-USP

- Prof. Dra. Ângela Marta Pereira das Dores Savioli - UEL 
Ao meu esposo Kazuto

e em especial aos meus filhos

Rafael e Camila. 


\section{Agradecimentos}

Ao Professor Flávio Ulhoa Coelho, por ser excelente orientador e ótima pessoa, que sempre esteve presente em todas as fases desse trabalho.

Aos meus amigos pelo incentivo, em especial ao Antônio Neri, Márcia Aguiar, Fernanda Estevam e Doris Pinheiro por sempre poder contar com eles.

Aos professores do IME que confiaram na conclusão deste trabalho.

Em especial a todos da minha família pela ajuda, compreensão e paciência que tiveram durante este período. 


\section{Resumo}

A Conjectura de Brauer-Thrall I foi resolvida por Roiter e estabelece que, se uma álgebra $\Lambda$ é de tipo de representação limitado, então $\Lambda$ é de tipo de representação finito. Esta conjectura atualmente é conhecida como Teorema de Roiter.

Seja $\Lambda$ uma álgebra de Artin de tipo de representação limitado.

O objetivo do trabalho é refazer a demonstração proposta por Gabriel para o Teorema de Roiter, utilizando conceitos e terminologias mais modernos como: cocobertura de categorias, categorias contravariantemente finitas e medida de Gabriel-Roiter. Tal medida leva em consideração a estrutura da possíveis filtrações por submódulos indecomponíveis de um dado módulo. Um resultado importante é o fato que o módulo indecomponível que possui a maior medida de Gabriel-Roiter ser o injetivo indecomponível de maior comprimento.

Seguindo a demonstração proposta por Gabriel, é feita uma partição na categoria dos módulos indecomponíveis explorando a noção dos injetivos relativos indecomponíveis. Utilizando o resultado de que se $\mathcal{C}$ é uma subcategoria de $i n d \Lambda$ tal que $\mathcal{C}$ tem uma cocobertura minimal finita $\mathcal{I}_{0}(\mathcal{C})$ (conjunto formado por todos os $\mathcal{C}$-injetivos indecomponíveis), então ind $\Lambda \backslash \mathcal{I}_{0}(\mathcal{C})$ também tem uma cocobertura finita. Usando o fato de que, como $\Lambda$ é de tipo de representação limitado e portanto existe apenas um número finito de medidas de Gabriel-Roiter, construímos uma partição de ind $\Lambda$ com apenas um número finito de subconjuntos finitos que a compõe. 


\section{Abstract}

The first Brauer-Thrall conjecture was proven by Roiter and it establishes that, if an algebra $\Lambda$ is of bounded representation type, then $\Lambda$ is of finite representation type. Nowadays, that conjecture is called Roiter's theorem.

Let $\Lambda$ be an Artin algebra of bounded representation type.

Our aim in this work is to present the proof proposed by Gabriel for Roiter's theorem, by using modern concepts and terminologies, such as cocover of categories, contravariantly finite and the so-called Gabriel-Roiter's measure. This measure takes into account the structure of possible filtrations by indecomposable submodules from a given module. The fact that the indecomposable module that has the biggest Gabriel-Roiter measure is the indecomposable injective of largest length has some nice consequences.

Following Gabriel's proof, we construct a partition inside the category of indecomposable modules, exploring the notion of indecomposable relative injectives. By assuming the result that if $\mathcal{C}$ is a subcategory of $i n d \Lambda$ such that $\mathcal{C}$ has a minimal finite cocover $\mathcal{I}_{0}(\mathcal{C})$ (set made of every indecomposable $\mathcal{C}$-injectives), then ind $\Lambda \backslash \mathcal{I}_{0}(\mathcal{C})$ has also a finite cocover. Thus using the fact that, as $\Lambda$ is of bounded representation type and therefore, that there exists only finite number of Gabriel-Roiter measures, one can construct a partition of $\Lambda$ with only finitely many layers. 


\section{Sumário}

0.1 Introdução . . . . . . . . . . . . . . . $\ldots$ iii

1 Preliminares 1

1.1 Teorema de Jordan-Hölder . . . . . . . . . . . . . . . . . . 1

1.2 Envolventes injetivas .................. 5

1.3 Seqüências de Auslander-Reiten . . . . . . . . . . . . . 6

1.4 Morfismos quase cindidos . . . . . . . . . . . . . . 7

2 Medida de Gabriel-Roiter 13

2.1 Relação de ordem em $\mathcal{P}_{f}\left(\mathbb{N}^{*}\right) \ldots \ldots \ldots \ldots \ldots$

2.2 Medida de Gabriel-Roiter . . . . . . . . . . . . . . . . 15

2.3 Propriedade Principal de Gabriel . . . . . . . . . . . . . . . . 26

3 Teorema de Roiter 32

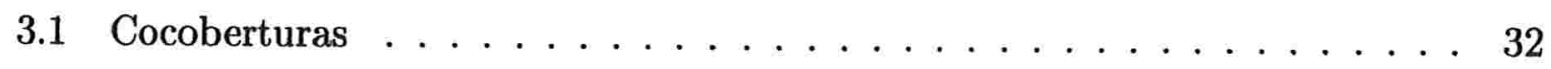

3.2 Subcategorias contravariantemente finitas . . . . . . . . . . . . 36

3.3 Teorema de Roiter . . . . . . . . . . . . . . . . . 43

3.4 Partição pré-injetiva . . . . . . . . . . . . . . . . 45

4 Medida de Gabriel-Roiter para a álgebra de Kronecker 51 
Referências Bibliográficas 


\subsection{Introdução}

Um dos problemas clássicos na Teoria de Anéis e Módulos que ajudaram a impulsionar a Teoria de Representações das Álgebras foi o de decidir a respeito das Conjecturas de Brauer-Thrall, BT-I e BT-II (ver abaixo). Essas conjecturas foram enunciadas pela primeira vez por Jans (em [Ja]), em 1957, mas acredita-se que elas sejam do início da década de 40.

Lembramos que uma álgebra $\Lambda$ é de tipo de representação limitado se existir um limite no comprimento das representações indecomponíveis e que $\Lambda$ é de tipo de representação finito se existir apenas um número finito de classes de isomorfismos de representações indecomponíveis. As Conjecturas de Brauer-Thrall são assim enunciadas:

BT-I Seja $\Lambda$ uma álgebra de dimensão finita sobre um corpo $k$. Se $\Lambda$ é de tipo de representação limitado, então é de tipo de representação finito.

BT-II Seja $k$ um corpo infinito e $\Lambda$ uma $k$-álgebra de tipo de representação infinito, então existe uma infinidade de dimensões para as quais existe um número infinito de $\Lambda$-módulos indecomponíveis não isomorfos com uma tal dimensão.

Em 1968, Roiter (em [Ro]) demonstrou a Conjectura de Brauer-Thrall I, atualmente conhecida como o Teorema de Roiter, para as álgebras de dimensão finita sobre um corpo algebricamente fechado. Em 1969, Yamagata (em [Ya]) propõe uma outra demonstração para essa conjectura para as álgebras de Artin, utilizando a chamada Teoria de Auslander-Reiten. Auslander, em 1974, estende o resultado para os Anéis de Artin [Au].

Gabriel (em [Ga]) apresenta uma reformulação da demonstração de Roiter para a Conjectura de Brauer-Thrall I, onde destaca a existência de um invariante que posteriormente será chamado, por Ringel [Ri], de medida de Gabriel-Roiter de um $\Lambda$-módulo. Tal medida leva em consideração, principalmente, a estrutura das possíveis filtrações por submódulos indecomponíveis de um dado módulo. A versão apresentada por Gabriel leva também em consideração a existência de uma partição na categoria de indecomponíveis de uma álgebra de tipo de representação limitado explorando a noção de injetivos relativos em subcategorias. Cabe mencionar aqui que essa última construção 
motivou Auslander e Smalø a introduzirem a noção de partição pré-injetiva (e também a. de partição pós-projetiva) em [AS] para uma álgebra de Artin arbitrária.

Recentemente, Ringel [Ri] observou que a suposição que Roiter e Gabriel fazem, desde o início, de que $\Lambda$ é de tipo de representação limitado, não é necessária e mostrou que o método exibido por Roiter e o invariante introduzido por Gabriel ajudam a esclarecer estrutura de categoria de $\Lambda$-módulos para uma álgebra de Artin arbitrária, especialmente para $\Lambda$ de tipo de representação infinito.

Nosso principal objetivo nesta dissertação é refazer a demonstração proposta por Gabriel para o Teorema de Roiter, utilizando conceitos e terminologias mais modernos como: contravariantemente finito (introduzido em [AS]) e medida de GabrielRoiter (introduzida em [Ri]). Utilizando esses conceitos, relembramos a definição de partição pré-injetiva [AS] para uma álgebra de Artin arbitrária.

O Capítulo 1 contém definições, notações e resultados básicos da categoria dos módulos finitamente gerados sobre uma álgebra de Artin, necessários para a compreensão dos capítulos que seguem.

O Capítulo 2 é dedicado ao estudo do invariante conhecido por medida de GabrielRoiter de um módulo. O capítulo foi baseado no artigo de Ringel [Ri], onde é definida a medida de Gabriel-Roiter de um $\Lambda$-módulo não nulo $M$ como sendo o máximo dos conjuntos $\left\{\ell\left(M_{1}\right), \ldots, \ell\left(M_{n}\right)\right\}$, onde $M_{1} \subset M_{2} \subset \ldots \subset M_{n}$ é uma cadeia de submódulos indecomponíveis de $M$ e $\ell\left(M_{i}\right)$ é o comprimento do módulo $M_{i}$. A ordem a ser utilizada na definição será introduzida no Capítulo 2. Neste capítulo temos o Teorema 2.3.1 que Ringel (em [Ri]) chama de Propriedade Principal de Gabriel, visto que muitos resultados úteis para o desenvolvimento do trabalho decorrem dele. Os resultados encontrados mostram que basta conhecermos a medida dos $\Lambda$-módulos indecomponíveis. Como trabalhamos com $\Lambda$-módulos finitamente gerados, todo $\Lambda$-módulo pode ser escrito como soma finita de módulos indecomponíveis e temos que $\mu\left(\oplus M_{i}\right)=\max \mu\left(M_{i}\right)$. E, ainda, que o módulo indecomponível que possui a maior medida de Gabriel-Roiter é o injetivo indecomponível de maior comprimento. Lembramos que os resultados encontrados no Capítulo 2 podem ser expandidos para os módulos não finitamente gerados (ver [Ri]).

No Capítulo 3, é apresentada a demonstração do Teorema de Roiter na versão de Gabriel, onde é feita uma partição na categoria de $i n d \Lambda$, sendo $\Lambda$ uma álgebra de tipo de 
representação limitado. Para tanto, lembramos a noção de partição pré-injetiva introduzida por Auslander e Smalø. Dada $\mathcal{C}$ uma subcategoria de ind $\Lambda$, podemos encontrar um subconjunto finito, formado por módulos $\mathcal{C}$-injetivos indecomponíveis (dizemos que um $\Lambda$-módulo $M$ é $\mathcal{C}$-injetivo se todo monomorfismo $f: M \longrightarrow Y$, com $Y \in a d d \mathcal{C}$, cinde). Esse conjunto formado por todos os $\mathcal{C}$-injetivos indecomponíveis é uma cocobertura minimal (dizemos que uma subcategoria $\mathcal{B} \subset \mathcal{C}$ é cocobertura minimal se para qualquer $X \in \mathcal{C}$ existe um monomorfismo $f: X \longrightarrow M^{\prime}$, com $M^{\prime} \in$ addC. Minimal porque nenhum subconjunto próprio de $\mathcal{B}$ tem essa propriedade). Denotamos por $\mathcal{I}_{0}(\mathcal{C})$ a subcategoria de $i n d \Lambda$ formada por todos os $\mathcal{C}$-injetivos indecomponíveis.

Observe que se $\mathcal{C}$ for uma subcategoria de $i n d \Lambda$ com cocobertura minimal finita $\mathcal{I}_{\mathbf{0}}(\mathcal{C})$, então ind $\Lambda \backslash \mathcal{I}_{0}(\mathcal{C})$ também tem uma cocobertura finita (ver Capítulo 3).

Utilizando-nos desse fato e lembrando que, como $\Lambda$ é de tipo de representação limitado, existe apenas um número finito de possíveis medidas de Gabriel-Roiter de $\Lambda$-módulos, construimos uma partição de ind $\Lambda$ com apenas um número finito de subconjuntos finitos que compõe esta partição. Na seção 3.4 , recordamos a definição de partição pré-injetiva e esquematizamos o resultado que garante a sua existência e unicidade. Como a partição que construimos para demonstrar o Teorema de Roiter satisfaz todas as condições dessa definição, a partição construída é de fato a partição pré-injetiva de ind $\Lambda$. Existe a noção dual de partição pré-injetiva, que é a partição pós-projetiva que pode ser encontrada em [AS].

No Capítulo 4, temos o cálculo de medida de Gabriel-Roiter dos módulos da álgebra de Kronecker, um exemplo de álgebra de tipo representação infinita, onde seus módulos apresentam uma estrutura bastante interessante e portanto suas medidas estão bem estabelecidas. 


\section{Capítulo 1}

\section{Preliminares}

Neste capítulo, iremos descrever alguns resultados e propriedades básicas da categoria dos módulos finitamente gerados sobre uma álgebra de Artin, que serão utilizados ao longo deste trabalho. Assumiremos muitos dos resultados aqui discutidos, sem explicitar suas demonstrações; no entanto, eles podem ser encontrados em detalhes em [A], [AF], [ARS], [CLS], [Co], [DK], [JM] e [Po].

\subsection{Teorema de Jordan-Hölder}

Um anel artiniano $\Lambda$ (com unidade) é uma álgebra de Artin se seu centro contiver um subanel artiniano sobre o qual $\Lambda$ é um módulo finitamente gerado. São exemplos de álgebras de Artin todas as álgebras de dimensão finita sobre um corpo.

Ao longo desse trabalho $k$ denotará um corpo algebricamente fechado.

Seja $\Lambda$ uma álgebra de Artin. Denotaremos por $\bmod \Lambda$ a categoria de todos os $\Lambda$-módulos à esquerda finitamente gerados. Neste trabalho, salvo menção explícita em contrário, todas as álgebras são de Artin e trabalharemos com módulos à esquerda finitamente gerados. E todas as subcategorias são plenas, a não ser em menção em contrário.

Todo módulo não nulo $M$ admite pelo menos dois submódulos: $M$ e $\{0\}$ (esses submódulos são conhecidos como submódulos triviais). Se $M$ não admite submódulos 
não triviais dizemos que $M$ é um módulo simples.

Um submódulo $N$ do módulo $M$ é dito ser maximal se $N \neq M$ e não existe submódulo $L$, diferente de $M$ e $N$ tal que $M \supset L \supset N$. Isso é equivalente a dizer que o quociente $\frac{M}{N}$ é um módulo simples.

Recordamos a seguir uma proposição necessária à demonstração do Teorema de JordanHölder, que pode ser encontrada em [AF].

Proposição 1.1.1. (Regra do paralelogramo) Sejam $N$ e P submódulos de um $\Lambda$-módulo M. Então tem-se que:

$$
\frac{N}{N \cap P} \cong \frac{N+P}{P}
$$

A relação entre os submódulos do enunciado pode-se visualizar no seguinte diagrama:

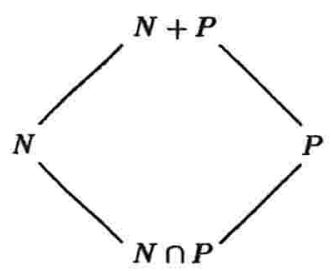

Continuamos agora com mais algumas definições necessárias.

A seqüência de submódulos de $M$,

$$
M=M_{0} \supset M_{1} \supset \ldots \supset M_{s}=0
$$

é chamada uma série de composição de $M$, se $\frac{M_{i}}{M_{i+1}}$ for um módulo simples, para $i=1, \ldots, s-1$.

Os módulos quocientes $\frac{M_{i}}{M_{i+1}}$ são chamados de fatores da série e o inteiro $s$ de comprimento da série.

Notemos que todo $\Lambda$-módulo $M$ admite uma série de composição. De fato, como $M$ é finitamente gerado, segue que o conjunto dos submódulos de $M$, diferentes do próprio $M$, contém um submódulo maximal $M_{1}$. Da mesma forma, ou $M_{1}=0$ ou $M_{1}$ contém submódulo maximal $M_{2}$. Repetindo o argumento, obtemos uma cadeia de submódulos de $M$ estritamente decrescente. Como $M$ é finitamente gerado, então ele é artiniano, 
isto é, toda cadeia descendente $M \supset M_{1} \supset M_{2} \supset \ldots \supset M_{n} \supset \ldots$ é estacionária. Logo a cadeia acima deve ser estacionária (isto é, para algum $n, M_{n}=0$ ). Assim, a cadeia obtida é uma série de composição.

$$
M \supset M_{1} \supset M_{2} \supset \ldots \supset M_{n}=0
$$

Provemos agora o Teorema de Jordan-Hölder.

\section{Teorema 1.1.2. Jordan-Hölder}

Se $M=M_{0} \supset M_{1} \supset \ldots \supset M_{s}=0 \quad$ e $\quad M=N_{0} \supset N_{1} \supset \ldots \supset N_{t}=0$ são duas séries de composição, então $s=t$ e existe $\sigma \in S_{n}$ tal que $\frac{M_{i-1}}{M_{i}} \cong \frac{N_{\sigma(i)-1}}{N_{\sigma(i)}}$.

Demonstração. Por indução sobre $s$. Se $s=1$, então o módulo $M=\frac{M_{0}}{M_{1}}$ é simples. Portanto, $t=1$ e $\frac{N_{0}}{N_{1}}=M=\frac{M_{0}}{M_{1}}$. Suponhamos $s>1$ e que para todas as séries de composição de comprimento $s-1$ o teorema é válido.

Se $M_{1}=N_{1}$, então $\frac{M_{0}}{M_{1}}=\frac{N_{0}}{N_{1}}$ e o teorema segue pela hipótese de indução.

Se $M_{1} \neq N_{1}$, então $M_{1}+N_{1} \neq M_{1}$, e como não existem submódulos intermediários entre $M$ e $M_{1}$ (pois $M_{1}$ é submódulo maximal de $M$ ), temos $M_{1}+N_{1}=M$ e, pela regra do paralelogramo

$$
\frac{M}{M_{1}}=\frac{M_{1}+N_{1}}{M_{1}} \cong \frac{N_{1}}{M_{1} \cap N_{1}} ; \quad \frac{M}{N_{1}}=\frac{M_{1}+N_{1}}{N_{1}} \cong \frac{M_{1}}{M_{1} \cap N_{1}}(*) .
$$

Segue, pela definição da série de composição, que $\frac{M}{M_{1}}$ e $\frac{M_{1}}{N_{1}}$ são simples. Logo, $\frac{N_{1}}{M_{1} \cap N_{1}}$ e $\frac{M_{1}}{M_{1} \cap N_{1}}$ são simples, o que equivale dizer $M_{1} \cap N_{1}$ é elemento maximal de $N_{1}$ e $M_{1}$.

Construimos uma série de composição para $M_{1} \cap N_{1}$ :

$$
M_{1} \cap N_{1}=L_{2} \supset L_{3} \supset \ldots \supset L_{k}=0
$$

Então $M_{1} \supset L_{2} \supset L_{3} \supset \ldots \supset L_{k}=0$ é uma série de composição de $M_{1}$. Comparando com a série $M_{1} \supset M_{2} \supset \ldots \supset M_{s}=0$, pela hipótese de indução, temos $s=k$ e existe uma bijeção tal que os fatores da série são dois a dois isomorfos.

Agora, compare as séries $M \supset M_{1} \supset L_{2} \supset L_{3} \supset L \supset \supset L_{s}=\begin{array}{llllll}0 & \supset\end{array}$ $M \supset N_{1} \supset L_{2} \supset L_{3} \supset \ldots \supset L_{s}=0$. Seus fatores coincidem a partir da terceira 
posição, e por (*) os fatores $\frac{M}{M_{1}} \cong \frac{N_{1}}{L_{2}}$ e $\frac{M}{N_{1}} \cong \frac{M_{1}}{L_{2}}$. Portanto, todos os fatores dessas séries são dois a dois isomorfos.

Finalmente, comparando as séries $N_{1} \supset L_{2} \supset \ldots \supset L_{s}=0$ e $N_{1} \supset N_{2} \supset \ldots \supset N_{t}=0$, pela hipótese de indução, temos $s=t$ e que os fatores destas séries são dois a dois isomorfos. E, assim, completamos a demonstração.

Logo, o comprimento de uma série de composição de um módulo $M$ é um invariante de $M$ e será chamado de comprimento de $M$, o qual denotaremos por $\ell(M)$, e aos fatores de uma tal série chamaremos de fatores de composição do módulo $M$. Também pelo Teorema de Jordan-Hölder, os fatores simples não dependem da escolha da série. Um $\Lambda$-módulo que contém uma série de composição é dito ser de comprimento finito. Como estamos trabalhando somente com módulos finitamente gerados, então podemos dizer que todo módulo tem comprimento finito.

Corolário 1.1.3. Se existe um epimorfismo $M \longrightarrow L$ com kernel $N$ (isto é, se um $\Lambda$-módulo $M$ é uma extensão de um módulo L por kernel $N$ ) então

$$
\ell(M)=\ell(L)+\ell(N) \text {. }
$$

Em particular, se $f: M \longrightarrow N$ é um morfismo, temos

$$
\ell(M)=\ell(\operatorname{Imf})+\ell(k e r f)
$$

Demonstração. Consideremos a série de composição do módulo $L \cong \frac{M}{N}$ : $L=L_{0} \supset L_{1} \supset \ldots \supset L_{k}=0$ e tomemos as pré-imagens $M_{i}$ de $L_{i}$ em $M$. Então, $\frac{M_{i}}{M_{i+1}} \cong \frac{L_{i}}{L_{i+1}}$ são módulos simples. Construamos uma série de composição do módulo $N$ : $N=N_{0} \supset N_{1} \supset \ldots \supset N_{t}=0$. Então

$$
M=M_{0} \supset M_{1} \supset \ldots \supset M_{k}=N=N_{0} \supset N_{1} \supset \ldots \supset N_{t}=0
$$

é uma série de composição do módulo $M$ de comprimento $k+t$, como queríamos.

\section{Corolário 1.1.4. (Regra de Grassmann)}

Se $L$ e $N$ são submódulos de $M$, então

$$
\ell(M+N)+\ell(L \cap N)=\ell(L)+\ell(N)
$$


Demonstração. Segue do corolário acima e da regra do paralelogramo.

\subsection{Envolventes injetivas}

Veremos agora módulos que apresentam como propriedade o fato de que todo submódulo é um somando direto.

Definição 1.2.1. Um $\Lambda$-módulo $M$ é semisimples se for soma direta de submódulos simples.

Mencionamos a seguinte caracterização dos módulos semisimples.

Proposição 1.2.2. São equivalentes:

(a) Mé semisimples.

(b) $M$ é soma de submódulos simples.

(c) Todo submódulo de $M$ é somando direto de $M$.

A prova desse resultado encontra-se em [ARS]. Cada $\Lambda$-módulo $M$ tem um submódulo semisimples maximal chamado de socle de $M$ (denotamos por $\operatorname{soc}(M)$ ). Como $\Lambda$ é artiniano, todo $\Lambda$-módulo $M$ não nulo tem submódulos simples, portanto $\operatorname{soc}(M) \neq 0$.

Para definir a envolvente injetiva de um módulo, precisamos recordar antes alguns outros conceitos.

Definição 1.2.3. Um $\Lambda$-módulo $I$ é injetivo se, dados $\Lambda$-módulos $M$ e $N$, um monomorfismo $f: M \longrightarrow N$ e um morfismo $g: M \longrightarrow I$, sempre existe um morfismo $h: N \longrightarrow I$ tal que $g=h \circ f$. De modo dual, um $\Lambda$-módulo $P$ é projetivo se, dados $\Lambda$-módulos $M$ e $N$, um epimorfismo $f: M \longrightarrow N$ e um morfismo $g: P \longrightarrow N$, sempre existe um morfismo $h: P \longrightarrow M$ tal que $g=f \circ h$.

Definição 1.2.4. Um monomorfismo $f: M^{\prime} \longrightarrow M$ em mod $\Lambda$ é chamado essencial se, dado um morfismo $g: M \rightarrow N$, tal que a composiçẫo $M^{\prime} \stackrel{f}{\rightarrow} M \stackrel{g}{\rightarrow} N$ seja um monomorfismo, segue que g é um monomorfismo. 
Definição 1.2.5. Sejam $\Lambda$-módulos $M$ e $I$. Um monomorfismo $f: M \longrightarrow I$ é uma envolvente injetiva de $M$ se $I$ é injetivo e f é essencial.

$\mathrm{O}$ próximo resultado garante a existência e unicidade de envolventes injetivas no contexto em que estamos trabalhado.

Lema 1.2.6. Seja $\Lambda$ uma álgebra de Artin. Todo $M \in$ mod $\Lambda$ tem uma envolvente injetiva única a menos de isomorfismos.

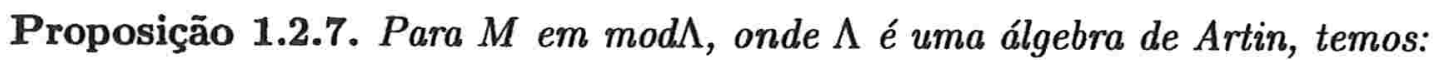

(a) $M=0$ se e somente se $\operatorname{soc}(M)=0$.

(b) Se $I, I^{\prime}$ são $\Lambda$-módulos injetivos, então $I \cong I^{\prime}$ se e somente $\operatorname{se} \operatorname{soc}(I)=\operatorname{soc}\left(I^{\prime}\right)$.

(c) Um injetivo I de mod $\Lambda$ é indecomponivel se e somente se soc(I) é simples.

Dizemos que o monomorfismo $f: M \longrightarrow N$ cinde se existir $f^{\prime}: N \longrightarrow M$ tal que $f^{\prime} \circ f=i d_{M}$. Analogamente, o epimorfismo $g: M \longrightarrow N$ cinde se existir $g^{\prime}: N \longrightarrow M$ tal que $g \circ g^{\prime}=i d_{N}$.

\subsection{Seqüências de Auslander-Reiten}

Para definir o quiver de Auslander-Reiten, precisamos das seqüências de AuslanderReiten e alguns outros conceitos que veremos a seguir. As demonstrações dos resultados desta seção podem ser encontradas em [ARS].

Definição 1.3.1. Uma seqüência exata curta é uma seqüência de módulos e morfismos

$$
0 \rightarrow L \stackrel{f}{\rightarrow} M \stackrel{g}{\rightarrow} N \rightarrow 0
$$

onde $f$ é um monomorfismo, $g$ é um epimorfismo e Imf $=$ kerg. Dizemos que a seqüência exata curta cinde se existir um morfismo $h: M \rightarrow L$ tal que $h \circ f=i d_{L}$ ou, equivalentemente, se existir um morfismo $h^{\prime}: N \rightarrow M$ tal que $g \circ h^{\prime}=i d_{N}$. 
Observação 1.3.2. Seja $0 \rightarrow L \stackrel{f}{\rightarrow} M \stackrel{g}{\rightarrow} N \rightarrow 0$ (*) uma seqüência exata curta.

(1) $S e(*)$ cinde, então $M \cong N \oplus L$;

(2) Se $N$ for um projetivo ou se $L$ for um injetivo, então (*) cindirá.

Definição 1.3.3. Uma seqüência exata curta que não cinde entre $\Lambda$-módulos $0 \rightarrow N \stackrel{f}{\rightarrow} E \stackrel{g}{\rightarrow} M \rightarrow 0$ é uma seqüência de Auslander-Reiten se:

(i) $N$ e $M$ são indecomponiveis;

(ii) para todo morfismo $h: X \longrightarrow M, X \in \bmod \Lambda$, que não seja um epimorfismo que cinde, existe $\phi: X \longrightarrow E$ tal que $g \phi=h$;

(ii') para todo morfismo $h: N \longrightarrow X, X \in \bmod \Lambda$, que não seja um monomorfismo que cinde, existe $\phi: E \longrightarrow X$ tal que $\phi f=h$.

Na realidade, as condições $(i i)$ e $\left(i i^{\prime}\right)$ são equivalentes (ver em [ARS]).

O próximo resultado mostra em que circunstâncias existem seqüências de AuslanderReiten.

Teorema 1.3.4. (Existência e Unicidade de seqüência de Auslander-Reiten) Seja $M$ um $\Lambda$-módulo indecomponível.

(1) Se $M$ não for projetivo, então existe e é única a seqüência de Auslander-Reiten terminando em $M: 0 \rightarrow N \stackrel{f}{\rightarrow} E \stackrel{g}{\rightarrow} M \rightarrow 0$.

(2) Se $M$ não for injetivo, então existe e é única a seqüência Auslander-Reiten começando em $M: 0 \rightarrow M \stackrel{f}{\rightarrow} E \stackrel{g}{\rightarrow} N \rightarrow 0$.

\subsection{Morfismos quase cindidos}

Vamos estudar os morfismos que compõem as seqüências de Auslander-Reiten a partir de sua propriedade de levantamento. As demonstrações dos resultados desta seção podem ser encontrados em [ARS] ou em [Co]. Ao longo deste trabalho todas as subcategorias são plenas. 
Definição 1.4.1. Se $M$ e $N$ são $\Lambda$-módulos, $\operatorname{seja~} \operatorname{rad}(M, N) \subset \operatorname{Hom}_{\Lambda}(M, N)$ o subconjunto de $\operatorname{Hom}_{\Lambda}(M, N)$ contendo todos os morfismos $f: M \longrightarrow N$ tais que para cada $L \in$ ind $\Lambda$, nenhuma composta do tipo $L \longrightarrow M \longrightarrow N \longrightarrow L$ é um isomorfismo. $O$ conjunto $\operatorname{rad}(M, N)$ resulta ser um subgrupo de $\operatorname{Hom}_{\Lambda}(M, N)$ e será chamado de radical de $\operatorname{Hom}_{\Lambda}(M, N)$.

A seguinte caracterização será bastante útil.

Proposição 1.4.2. Seja $M \in i n d \Lambda$.

(a) Seja $f: N \rightarrow M$ um morfismo. Então $f \in \operatorname{rad}(N, M)$ se e só se $f$ não é um epimorfismo que cinde.

(b) Seja $g: M \rightarrow N$ um morfismo. Então $g \in \operatorname{rad}(M, N)$ se e só se $g$ não é um monomorfismo que cinde.

Com a definição de radical de $\operatorname{Hom}_{\Lambda}(N, M)$ podemos definir os morfismos quase cindidos à direita.

Definição 1.4.3. Sejam $N \in$ ind $\Lambda$ e um morfismo que não cinde $f: M \longrightarrow N$. Dizemos que $f$ é um morfismo quase cindido à direita se $(X, f): \operatorname{Hom}_{\Lambda}(X, M) \longrightarrow \operatorname{Hom}_{\Lambda}(X, N)$ onde $g \longmapsto f \circ g$ tem imagem $\operatorname{rad}(X, N)$ para todo $X \in \bmod \Lambda$. Isso equivale a dizer que o diagrama abaixo comuta, isto é, a cada $h: X \longrightarrow N$ que não é epimorfismo que cinde, existe $g: X \longrightarrow M$ tal que $h=f \circ g$.

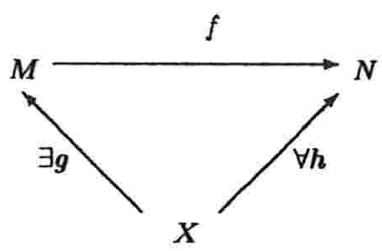

De maneira similar, dizemos que $f: N \longrightarrow M$ é um morfismo quase cindido à esquerda se $(f, Y): \operatorname{Hom}_{\Lambda}(M, Y) \longrightarrow \operatorname{Hom}_{\Lambda}(N, Y)$, onde $g \mapsto g \circ f$, tem imagem $\operatorname{rad}(N, Y)$ para todo $Y \in \bmod \Lambda$.

Um morfismo quase cindido à direita (respectivamente à esquerda) $f$ é minimal se $h \circ f=f$ (respectivamente $h \circ f=f$ ) para algum morfismo $h$, então $h$ é um isomorfismo. 
Observação 1.4.4. O morfismo quase cindido à direita minimal e o morfismo quase cindido à esquerda minimal são também conhecidos como morfismo poço e morfismo fonte, respectivamente.

Lema 1.4.5. Se $0 \rightarrow N \stackrel{f}{\rightarrow} E \stackrel{g}{\rightarrow} M \rightarrow 0$ é uma seqüência de Auslander-Reiten, então

1. $f$ é um morfismo quase cindido à esquerda.

2. $g$ é um morfismo quase cindido à direita.

Corolário 1.4.6. Seja $M \in$ ind $\Lambda$.

1. Se $M$ não é projetivo e se $g: E \rightarrow M$ é um morfismo quase cindido à direita, então $g$ é um epimorfismo e $0 \rightarrow \operatorname{kerg} \stackrel{\iota}{\hookrightarrow} E \stackrel{g}{\rightarrow} M \rightarrow 0$ é uma seqüência de Auslander-Reiten.

2. Se $M$ não é injetivo e se $f: M \rightarrow E$ é um morfismo quase cindido à esquerda, então $f$ é um monomorfismo e $0 \rightarrow M \stackrel{f}{\rightarrow} E \stackrel{\pi}{\rightarrow}$ coker $f \rightarrow 0$ é uma seqüência de Auslander-Reiten.

Exemplo 1.4.7. A inclusão natural $\iota: \operatorname{radP} \hookrightarrow P$ é quase cindida à direita para todo módulo projetivo indecomponível não simples $P$.

Definição 1.4.8. Seja $\mathcal{C}$ uma subcategoria de mod $\Lambda$. Dizemos que $\mathcal{C}$ tem morfismos quase cindidos à direita se para todo $M \in$ indC existir um morfismo quase cindido $\grave{a}$ direita $f: N \longrightarrow M$ com $N \in$ addC.

Notemos que o morfismo acima $f$ pertence ao radical $\operatorname{rad}(N, M)$, pois segue, da definição de morfismo quase cindido à direita, que $f$ é um morfismo que não cinde.

Agora, veremos que alguns morfismos quase cindido (à direita ou à esquerda) possuem certas propriedades de irredutibilidade no sentido dado pela seguinte definição.

Definição 1.4.9. Um morfismo que não cinde $f \in \operatorname{Hom}_{\Lambda}(X, Y) \operatorname{com} X, Y$ em $\bmod \Lambda$ é irredutível se, para cada decomposição $f=g h$, ou $g$ é um epimorfismo que cinde ou $h$ é um monomorfismo que cinde. 
Observação 1.4.10. Se $f: X \rightarrow Y$ é irredutivel, então $f$ é um epimorfismo ou um monomorfismo. De fato, dado qualquer morfismo $f: X \rightarrow Y$, podemos decompô-lo naturalmente como $f=g h$, onde $h: X \rightarrow \operatorname{Imf}$ é dado por $h(x)=f(x)$ para $x \in X$ e $g$ é a inclusão natural de Imf em $Y$. Se f for irredutível, então ou $g$ é um epimorfismo que cinde e, portanto, $f$ é um epimorfismo ou $h$ é um monomorfismo que cinde e f é, portanto, um monomorfismo.

A relação entre morfismo irredutível e morfismo quase cindido à direita (e à esquerda) é dada pela seguinte proposição.

Proposição 1.4.11. Sejam $M, N$ dois $\Lambda$-módulos, com $M$ indecomponível.

1. Um morfismo $f: N \longrightarrow M$ é irredutível se e somente se existe um morfismo $f^{\prime}: N^{\prime} \longrightarrow M$ tal que o morfismo induzido $\left(f, f^{\prime}\right): N \oplus N^{\prime} \longrightarrow M$ é um morfismo quase cindido à direita minimal.

2. Um morfismo $f: M \longrightarrow N$ é irredutível se e somente se existe um morfismo $f^{\prime}: M \longrightarrow N^{\prime}$ tal que o morfismo induzido $\left(f, f^{\prime}\right)^{t}: M \longrightarrow N \oplus N^{\prime}$ é um morfismo quase cindido à esquerda minimal.

Uma álgebra $\Lambda$ e seus módulos podem ser representados em termos de certas estruturas chamadas de quivers, que são grafos orientados. Vejamos a seguinte definição.

Definição 1.4.12. Um quiver $Q$ é dada por dois conjuntos $Q_{0}$ e $Q_{1}$ e um par de funçôes $s, e: Q_{1} \longrightarrow Q_{0}$. Os elementos de $Q_{0}$ são chamados de vértices de $Q$ enquanto que os de $Q_{1}$ são as flechas de $Q$. Dada uma flecha $\alpha \in Q_{1}, s(\alpha)$ é o vértice inicial de $\alpha$ enquanto que e $(\alpha)$ é o vértice final de $\alpha$.

Seja $\Lambda$ uma $k$-álgebra de dimensão finita onde $k$ é um corpo algebricamente fechado. Vamos agora associar, a $\Lambda$, um quiver $\Gamma_{\Lambda}$, onde os vértices e as flechas representarão, respectivamente, os $\Lambda$-módulos indecomponíveis e os morfismos irredutíveis entre eles. Tal quiver $\Gamma_{\Lambda}$ é chamado de quiver de Auslander-Reiten e foi formalizado por Ringel. Lembramos que tal definição pode ser feita mais geralmente para álgebra de Artin mas não a discutiremos aqui (ver [ARS] para detalhes). 
Definição 1.4.13. O quiver de Auslander-Reiten de $\Lambda$ é o quiver $\Gamma_{\Lambda}$ definido como segue:

(1) Os vértices estão em correspondência biunivoca com as classes de isomorfismos dos $\Lambda$-módulos indecomponíveis, isto é, para cada $M \in$ ind $\Lambda$, associamos um vértice $[M]$ e os vértices $[M]$ e $\left[M^{\prime}\right]$ são os mesmos se e somente se $M \cong M^{\prime}$.

(2) Dados $M, M^{\prime} \in$ ind $\Lambda$, o número de flechas $[M] \rightarrow\left[M^{\prime}\right]$ é igual à dimensão do $k$-espaço vetorial $\frac{\operatorname{rad}\left(M, M^{\prime}\right)}{\operatorname{rad}^{2}\left(M, M^{\prime}\right)}$.

Seja $M$ um $\Lambda$-módulo indecomponível. Se $M$ não for projetivo os morfismos irredutíveis chegando em $M$ podem ser obtidos a partir das seqüências de AuslanderReiten que terminam em $M$. Caso o módulo $M$ seja um projetivo indecomponível, os morfismos irredutíveis chegando em $M$ são, a menos de isomorfismo, as inclusões dos somandos diretos do $\operatorname{rad} M$ em $M$. Dualmente, os morfismos irredutíveis saindo de $M$ podem ser obtidas a partir das seqüências de Auslander-Reiten. Caso $M$ seja um injetivo indecomponível, os morfismos irredutíveis saindo de $M$ são, a menos de isomorfismo, as projeções canônicas $M$ sobre os somandos $\operatorname{diretos} \frac{M}{\operatorname{soc} M}$.

A seguir veremos um exemplo de quiver de Auslander-Reiten de uma álgebra.

Exemplo 1.4.14. Consideremos a álgebra $\Lambda=\frac{k \Delta}{I}$, onde $\Delta$ é o quiver

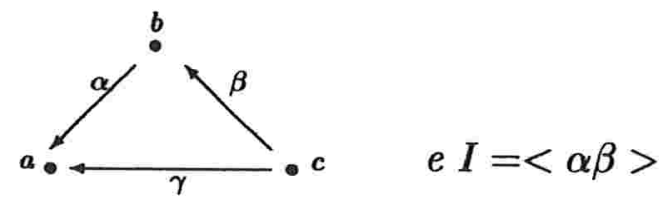

O quiver de Auslander-Reiten $\Gamma_{\Lambda}$ é:

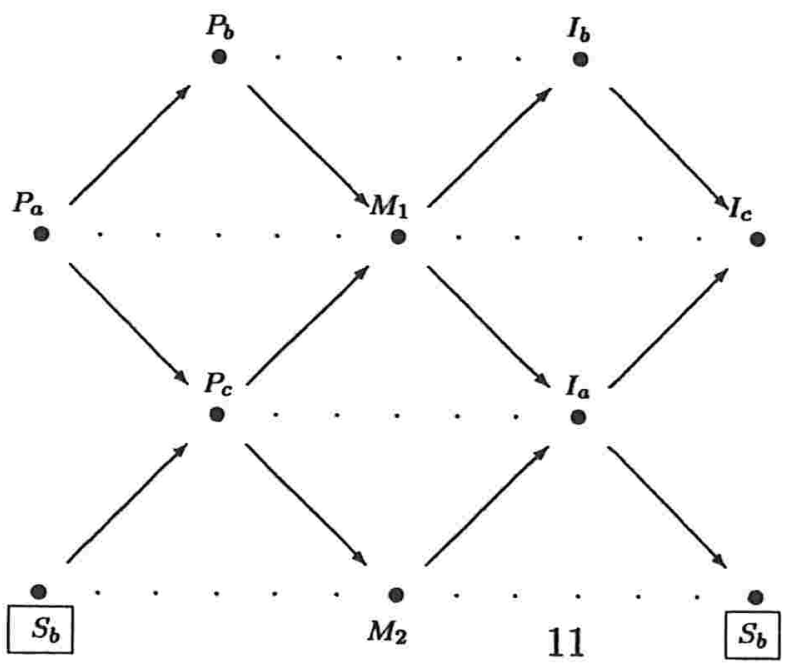


onde identificamos as duas cópias de $S_{b}$.

Representamos os módulos indecomponíveis como sendo:

$P_{a}: \stackrel{a}{\bullet}$<smiles>[10BH][10BH]</smiles><smiles>[12CH3][13CH3]</smiles>

$S_{b}: \stackrel{b}{\bullet}$

$M_{1}$ :

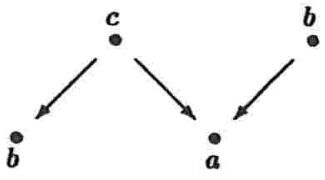

$M_{2}:$

$I_{a}:$

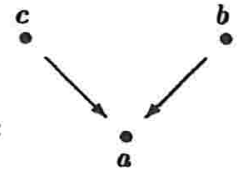

$I_{b}: \stackrel{c}{b}$

$I_{c}:{ }^{c}$ 


\section{Capítulo 2}

\section{Medida de Gabriel-Roiter}

Este capítulo é dedicado ao estudo do invariante introduzido por Gabriel em [Ga] para explicar o método utilizado por Roiter [Ro] na demonstração da primeira Conjectura de Brauer-Thrall (a qual é atualmente conhecida por Teorema de Roiter). Muitos dos resultados aqui presentes foram utilizados por Ringel [Ri] inspirado no método utilizado por Roiter na sua demonstração e pela reformulação dada por Gabriel. Ringel chama esse invariante de medida de Gabriel-Roiter. Nesse trabalho, Ringel observa que a suposição que tanto Roiter quanto Gabriel fazem de início, assumindo que a álgebra é de tipo de representação limitado, não é necessária. Ele também mostra que o método utilizado por Roiter e o invariante introduzido por Gabriel ajudam a decifrar a estrutura da categoria dos $\Lambda$-módulos para uma álgebra de Artin $\Lambda$ qualquer, independentemente de seu tipo de representação.

Como o objetivo de nosso trabalho é refazer a demonstração proposta por Gabriel para o Teorema de Roiter, então vamos, em algum momento, também assumir que a álgebra é de tipo de representação limitado. Lembramos, no entanto, que todos os resultados aqui apresentados podem ser generalizados para módulos não finitamente gerados. Essas generalizações podem ser encontradas em [Ri].

A medida de Gabriel-Roiter leva em consideração, principalmente, a estrutura das possíveis filtrações por submódulos indecomponíveis de um dado $\Lambda$-módulo $M$. Veremos que a medida de Gabriel-Roiter é um subconjunto finito de $\mathbb{N}^{*}$. Essa medida pode ser também considerada como um número racional entre 0 e 1 , que depende apenas dos 
comprimentos dos submódulos indecomponíveis de $M$.

\subsection{Relação de ordem em $\mathcal{P}_{f}\left(\mathbb{N}^{*}\right)$}

Vamos definir, nesta seção, uma relação de ordem que será utilizada para a chamada medida de Gabriel-Roiter.

Sejam $\mathbb{N}^{*}$ o conjunto dos números naturais não nulos e $\mathcal{P}_{f}\left(\mathbb{N}^{*}\right)$ o conjunto de todos os subconjuntos finitos de $\mathbb{N}^{*}$. Para $a, b \in \mathbb{N}^{*}, a \leq b$, denotamos $[a, b]_{\mathbb{N}^{*}}=\left\{n \in \mathbb{N}^{*} \mid a \leq n \leq b\right\}$. Consideremos a relação de ordem total: se $I, J$ são dois subconjuntos finitos diferentes de $\mathbb{N}^{*}$, escrevemos $I<J$ se o menor elemento de $(I \backslash J) \cup(J \backslash I)$ pertencer a $J$ (isto é, se existe um número natural não nulo $a \in(J \backslash I)$ e $I \cap[1, a-1]_{\mathbb{N}^{*}}=J \cap[1, a-1]_{\mathbb{N}^{*}}$ e por convenção $\left.[1,0]_{\mathbb{N}^{*}}=\emptyset\right)$. Escrevemos $I \leq J$ se $I<J$ ou $I=J$.

Dizemos $I \ll J$ se $I \subset J$ e para todos elementos $a \in I$ e $b \in J \backslash I$, temos $a<b$. Dizemos que $J$ começa com $I$ se $I=J$ ou $I \ll J$.

Exemplo 2.1.1. Vamos ordenar todos os subconjuntos de $\{1,2,3,4\}$ com relação a essa ordem acima:

$\{4\}<\{3\}<\{3,4\}<\{2\}<\{2,4\}<\{2,3\}<\{2,3,4\}<\{1\}<\{1,4\}<\{1,3\}<$ $\{1,3,4\}<\{1,2\}<\{1,2,4\}<\{1,2,3\}<\{1,2,3,4\}$.

Observemos que $\{1,2,4\}$ começa com $\{1\}$, com $\{1,2\}$ ou com $\{1,2,4\}$.

Observação 2.1.2. Seja $m \in \mathbb{N}^{*}$. Observemos que, no subconjunto $[1, m]_{\mathbb{N}^{*}} \subseteq \mathbb{N}^{*}$ o elemento minimal é $\{m\}$ e o elemento maximal é $[1, m]_{\mathbb{N}^{*}}$.

Lema 2.1.3. Sejam $I, J, L$ subconjuntos finitos de $\mathbb{N}^{*}$.

(a) $S e I \subseteq J$, então $I \leq J$.

(b) $S e I \leq J \leq L$ e L começa com $I$, então $J$ começa com $I$.

(c) Sejam $m(I), m(J)$, respectivamente, os elementos maximais dos conjuntos $I$ e $J$. Se $I \leq J$ e $J \backslash\{m(J)\}<I$, então $m(I) \geq m(J)$. 
Demonstração. (a) Se $I=J$, não há nada a provar. Suponha que $I \neq J$ e como $I \subseteq J$ temos $I \backslash J=\emptyset$ e $J \backslash I \neq \emptyset$. Como $(I \backslash J) \cup(J \backslash I)=(J \backslash I)$, segue que $I \leq J$.

(b) Se $I=L$ então temos $I=J=L$, nesse caso o resultado está provado. Se $I \neq J$, como $I \leq J$, então existe $c \neq 0, c \in J \backslash I$ tal que $[1, c-1]_{\mathbb{N}^{*}} \cap I=[1, c-1]_{\mathbb{N}^{*}} \cap J$. Por outro lado, temos que $J \leq L$ de onde segue que existe $b \in \mathbb{N}^{*}, b \in L \backslash J$ que é o menor elemento de $(J \backslash L) \cup(L \backslash J)$.

Podemos ter dois casos:

Se $c \leq b$ então, $c \in L$ (pois se $c \notin L$, o menor elemento de $(L \backslash J) \cup(J \backslash L)$ seria $c$ e então teríamos $L<J$, absurdo.), e como $L$ comę̧a com $I$ e $c \in L \backslash I$, então para todo $i \in I$, temos $i<c$. Mas $c$ é o menor elemento de $J \backslash I$, isto é, para todo $j \in J \backslash I, c \leq j$, logo $i<j$, para todo $i \in I$ e $j \in J \backslash I$. Portanto, neste caso, $J$ começa com $I$.

Se $c>b$ temos que $b \notin I$ (pois se $b \in I$, teríamos $b \in I \backslash J$ e $b<c$, logo $J<I$, absurdo.), e como $L$ começa com $I$, para todo $i \in I, i<b$, pois $b \in L \backslash I$, e ainda $b<c$ então para todo $i \in I$, temos $i<c$. Em particular $c$ é o menor elemento de $J \backslash I$, então, para todo $j \in J \backslash I, i<c<j$. Logo $I=I \cap[1, c-1]_{\mathbb{N}^{*}}=J \cap[1, c-1]_{\mathbb{N}^{*}}$, isto é, $I \subset J$. Portanto, $J$ começa com $I$.

(c) Como $I \leq J$, seja $a \in \mathbb{N}^{*}$ tal que $a \in J \backslash I$ e $J \cap[1, a-1]_{\mathbb{N}^{*}}=I \cap[1, a-1]_{\mathbb{N}^{*}}$, isto é, o menor elemento de $(J \backslash I) \cup(I \backslash J)$ pertence a $J$. Notemos que $a \leq m(J)$, pois $a \in J$ e $m(J)$ é o elemento maximal de $J$. Suponhamos que $a<m(J)$, nesse caso

$$
(J \backslash\{m(J)\}) \cap[1, a-1]_{\mathbb{N}^{*}}=J \cap[1, a-1]_{\mathbb{N}^{*}}=I \cap[1, a-1]_{\mathbb{N}^{*}},
$$

e $a \in(J \backslash\{m(J)\}) \backslash I$, o que contradiz a hipótese $(J \backslash\{m(J)\}<I)$. Logo, $a=m(J)$ de onde segue que $m(I) \geq m(J)$ (pois se $m(I)<m(J)=a$, então $m(I) \in[1, a-1]_{\mathbb{N}^{*}} \cap I=J \cap[1, a-1]_{\mathbb{N}^{*}}$, de onde segue que $J \backslash\{m(J)\}=I$. O que é um absurdo, pois $J \backslash\{m(J)\}<J)$.

\subsection{Medida de Gabriel-Roiter}

A partir de agora, $\Lambda$ denotará uma álgebra de Artin. Utilizando a relação de ordem acima, vamos definir a medida de Gabriel-Roiter para um $\Lambda$-módulo $M$. Veremos que, para os módulos indecomponíveis que apresentam socle simples, a medida de Gabriel- 
Roiter é facilmente calculada, bastando conhecer o seu comprimento. E ainda, que a medida de Gabriel-Roiter pode ser considerada um número racional entre 0 e 1.

Definição 2.2.1. Para um $\Lambda$-módulo não nulo $M$, definimos a medida de Gabriel-Roiter $\mu(M)$ como o máximo dos conjuntos $\left\{\ell\left(M_{1}\right), \ldots, \ell\left(M_{n}\right)\right\}$ com a relação de ordem definida acima, onde $M_{1} \subset M_{2} \subset \ldots \subset M_{n}$ é uma cadeia de submódulos indecomponíveis de $M$; se $M$ é o módulo nulo, convencionamos que, $\mu(M)=\emptyset$, isto é,

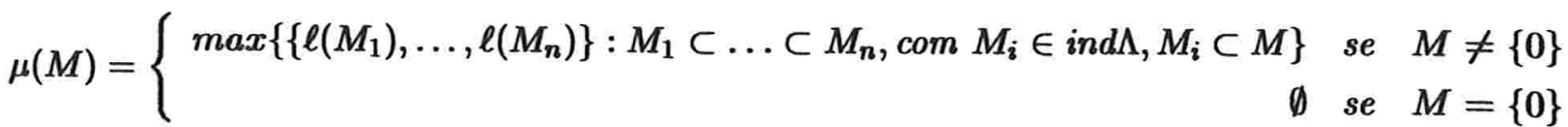

Observação 2.2.2. Seguem da definição os seguintes fatos:

(a) Se $M^{\prime}$ é submódulo de $M$, então $\mu\left(M^{\prime}\right) \leq \mu(M)$.

(b) Para todo $\Lambda$-módulo $M$, a medida de Gabriel-Roiter $\mu(M)$ é o máximo das medidas $\mu\left(M^{\prime}\right)$, onde $M^{\prime}$ é um submódulo indecomponível de $M$.

(c) Sejam $M$ e $N$ dois $\Lambda$-módulos indecomponíveis tais que $\mu(M)=\mu(N)$, então $\ell(M)=\ell(N)$.

Veremos a seguir alguns exemplos de medida de Gabriel-Roiter. A partir de agora, denotamos por $S_{i}, P_{i}$ e $I_{i}$, respectivamente, o módulo simples, projetivo e injetivo para o vértice $i$.

Exemplo 2.2.3. Seja a $k$-álgebra $\Lambda=k \Delta$ dada pelo quiver $\Delta$ :

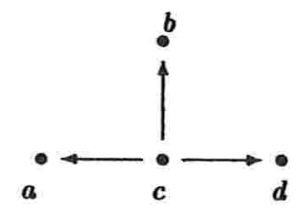

$\mathrm{O}$ quiver de Auslander-Reiten $\Gamma_{\Lambda}$ é:

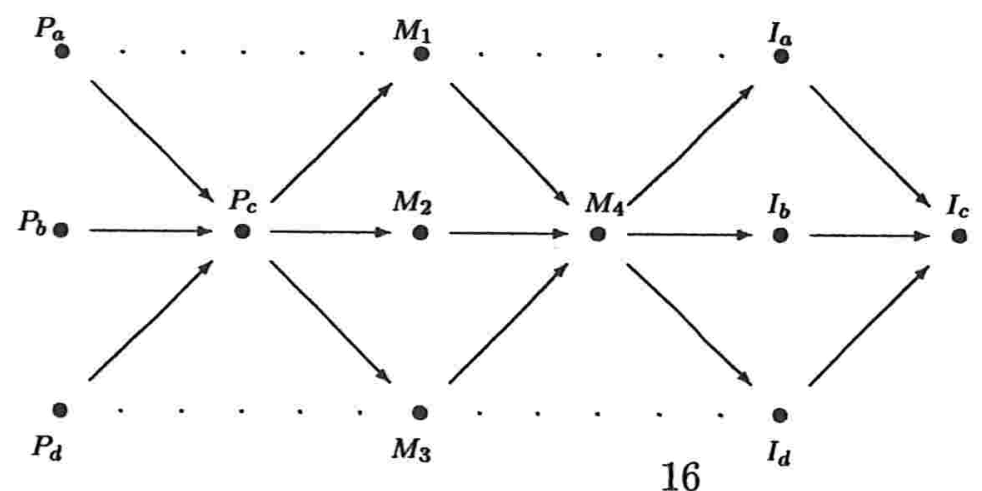


Os $\Lambda$-módulos indecomponíveis são representados como sendo:
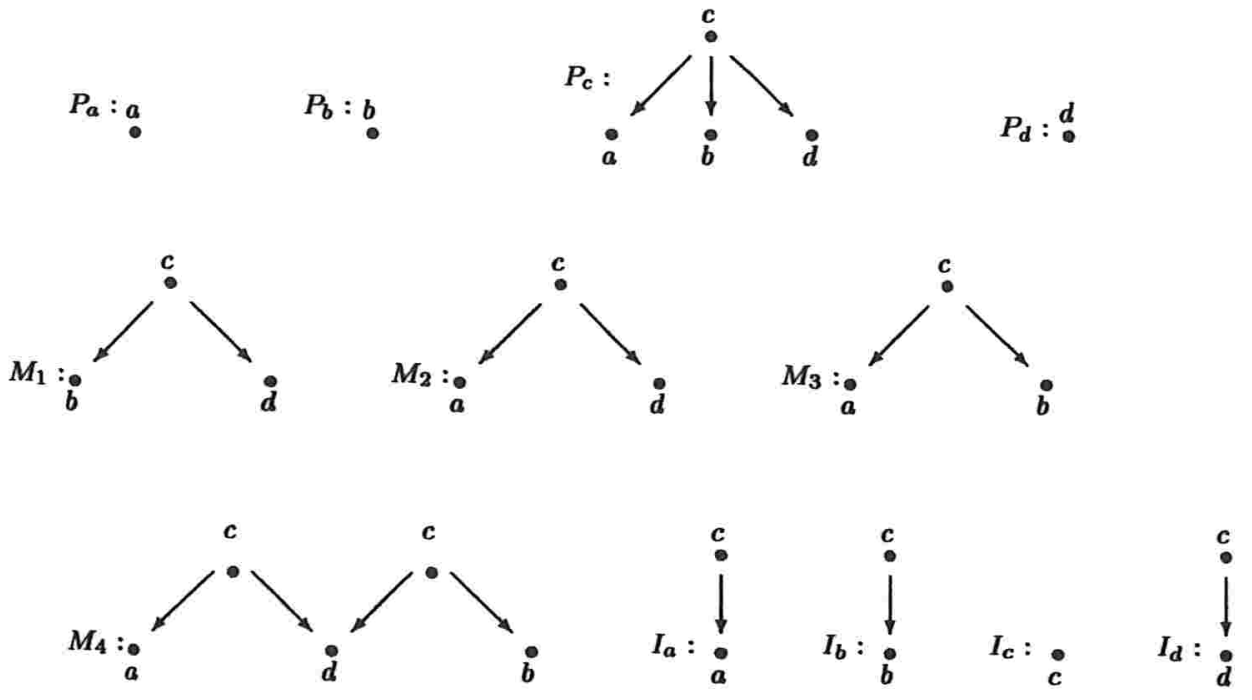

Nesta álgebra, os módulos acima têm as seguintes medidas de Gabriel-Roiter:

$$
\begin{aligned}
& \mu\left(P_{a}\right)=\mu\left(P_{b}\right)=\mu\left(P_{d}\right)=\mu\left(I_{c}\right)=\{1\} \\
& \mu\left(I_{a}\right)=\mu\left(I_{b}\right)=\mu\left(I_{d}\right)=\{1,2\} \\
& \mu\left(M_{1}\right)=\mu\left(M_{2}\right)=\mu\left(M_{3}\right)=\{1,3\} \\
& \mu\left(P_{c}\right)=\{1,4\} \quad e \\
& \mu\left(M_{4}\right)=\{1,3,5\} .
\end{aligned}
$$

E temos a ordenação:

$$
\{1\}<\{1,4\}<\{1,3\}<\{1,3,5\}<\{1,2\} \text {. }
$$

Exemplo 2.2.4. Considere a álgebra $\Lambda=\frac{k \Delta}{I}$, onde $\Delta$ é o quiver

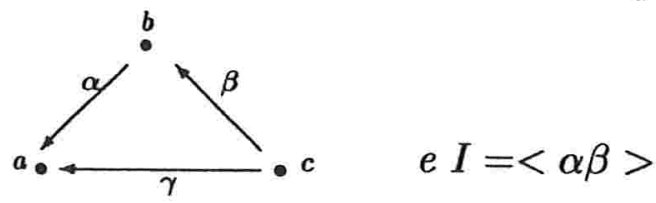

$\mathrm{O}$ quiver de Auslander-Reiten $\Gamma_{\Lambda}$ é: 


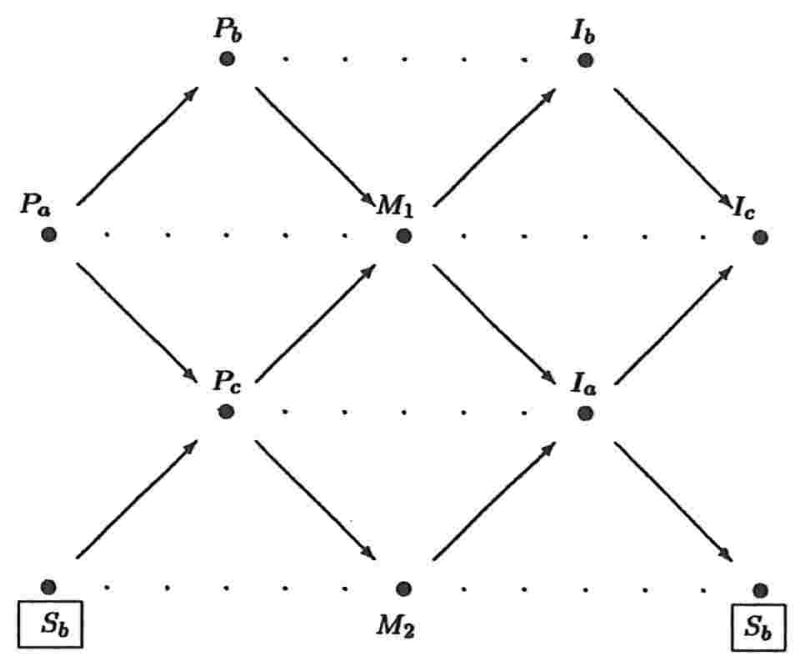

onde identificamos as duas cópias de $S_{b}$.

Representamos os módulos indecomponíveis como sendo:

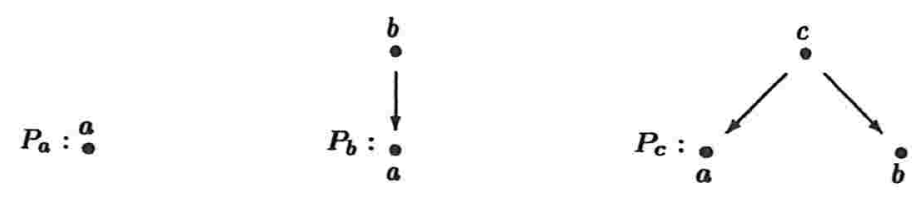

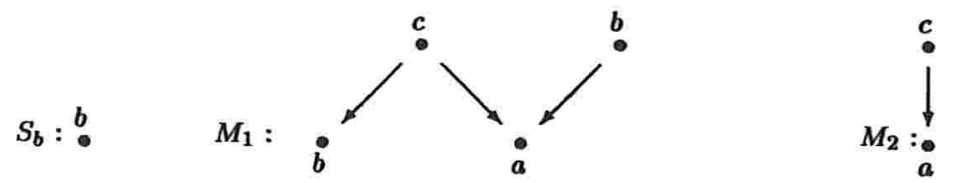

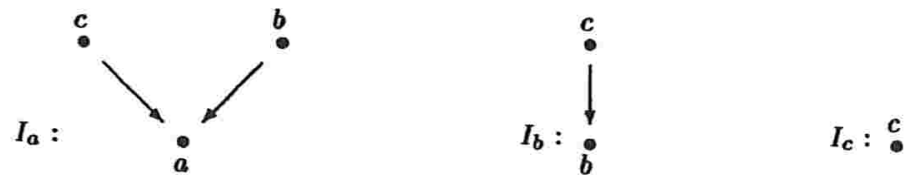

Temos, assim, as medidas de Gabriel-Roiter dos $\Lambda$-módulos indecomponíveis:

$$
\begin{aligned}
& \mu\left(P_{a}\right)=\mu\left(S_{b}\right)=\mu\left(I_{c}\right)=\{1\} \\
& \mu\left(P_{b}\right)=\mu\left(M_{2}\right)=\mu\left(I_{b}\right)=\{1,2\} \\
& \mu\left(P_{c}\right)=\{1,3\}
\end{aligned}
$$




$$
\mu\left(I_{a}\right)=\{1,2,3\}
$$

$\mu\left(M_{1}\right)=\{1,2,4\}$. Observe que existem três cadeias de submódulos indecomponíveis para $M_{1}: S_{a} \subset P_{b} \subset M_{1}, S_{b} \subset P_{c} \subset M_{1}$ e $S_{a} \subset P_{c} \subset M_{1}$. Da primeira cadeia, temos $\{1,2,4\}$ e das segunda e terceira, temos $\{1,3,4\}$. Como $\{1,3,4\}<\{1,2,4\}$, segue que $\mu\left(M_{1}\right)=\{1,2,4\}$.

E temos:

$$
\{1\}<\{1,3\}<\{1,2\}<\{1,2,4\}<\{1,2,3\} .
$$

Exemplo 2.2.5. Considere a $k$-álgebra $\Lambda=k \Delta$, onde $\Delta$ é o quiver:

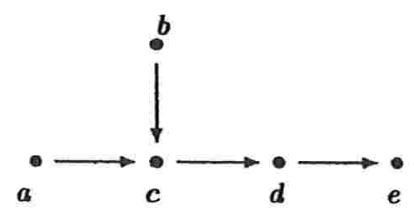

O quiver de Auslander-Reiten $\Gamma_{\Lambda}$ é:

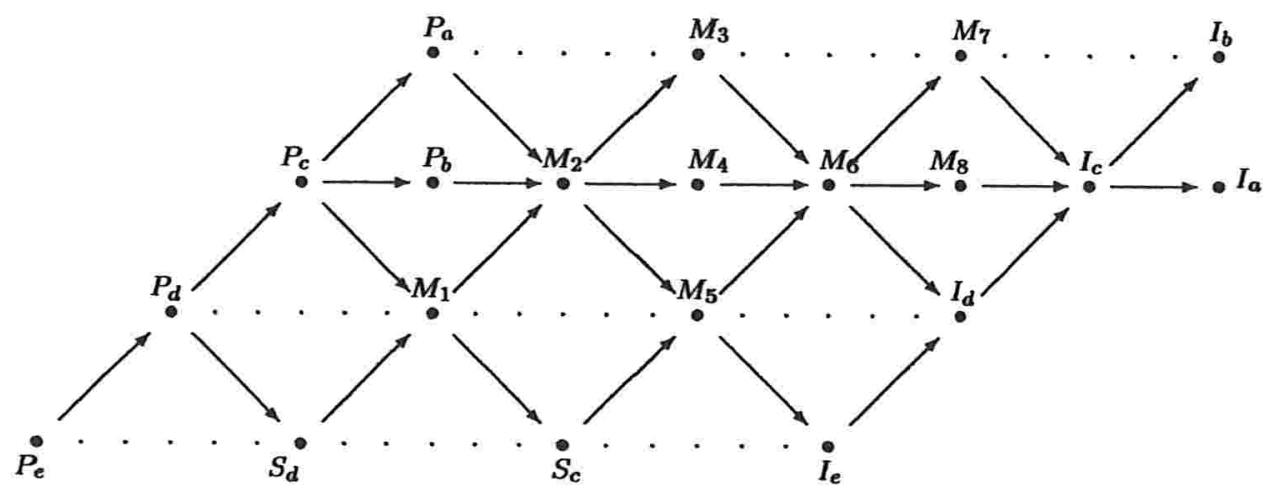

Representamos os módulos indecomponíveis como sendo:

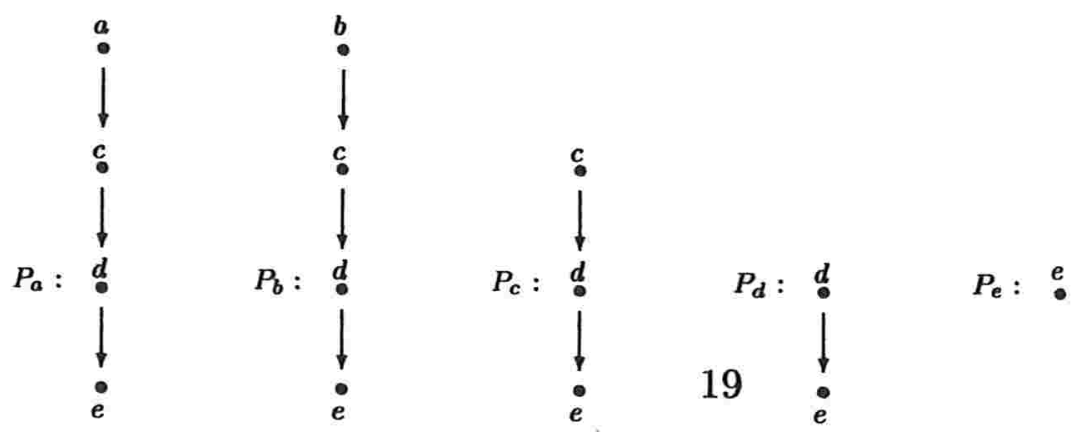



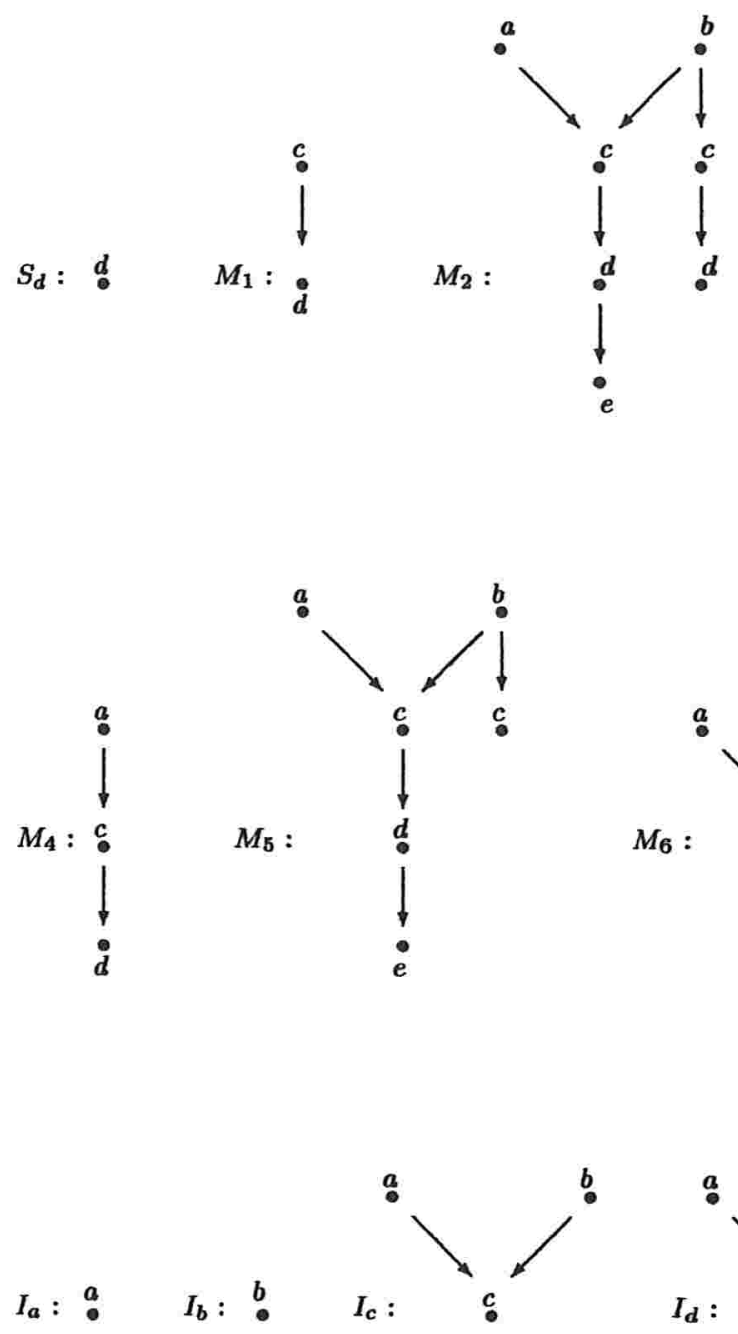
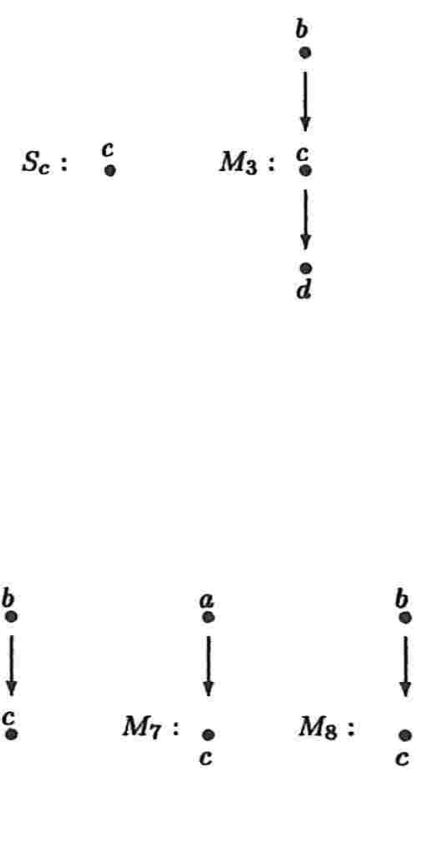
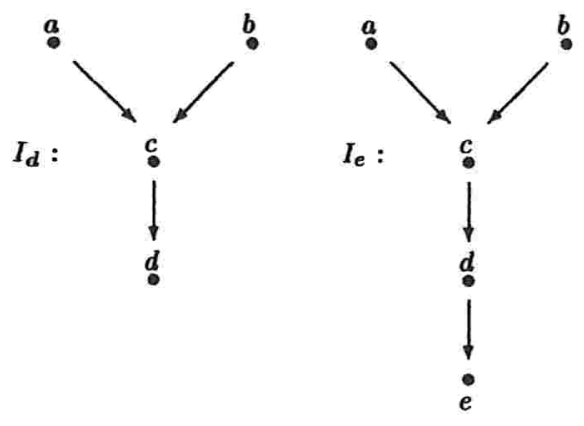

As medidas de Gabriel-Roiter dos módulos indecomponíveis da álgebra $\Lambda$ são dadas por:

$$
\begin{aligned}
& \mu\left(P_{e}\right)=\mu\left(S_{d}\right)=\mu\left(S_{c}\right)=\mu\left(I_{a}\right)=\mu\left(I_{b}\right)=\{1\} \\
& \mu\left(P_{d}\right)=\mu\left(M_{1}\right)=\mu\left(M_{7}\right)=\mu\left(M_{8}\right)=\{1,2\} \\
& \mu\left(P_{c}\right)=\mu\left(M_{3}\right)=\mu\left(M_{4}\right)=\mu\left(I_{c}\right)=\{1,2,3\}
\end{aligned}
$$




$$
\begin{aligned}
& \mu\left(P_{b}\right)=\mu\left(P_{a}\right)=\mu\left(I_{d}\right)=\{1,2,3,4\} \\
& \mu\left(M_{6}\right)=\{1,2,3,5\} \\
& \mu\left(I_{e}\right)=\{1,2,3,4,5\} \\
& \mu\left(M_{5}\right)=\{1,2,3,4,6\} \\
& \mu\left(M_{2}\right)=\{1,2,3,4,7\}
\end{aligned}
$$

Temos:

$\{1\}<\{1,2\}<\{1,2,3\}<\{1,2,3,5\}<\{1,2,3,4\}<\{1,2,3,4,7\}<\{1,2,3,4,6\}<$ $\{1,2,3,4,5\}$.

Mostraremos a seguir algumas relações que podem existir entre a medida de GabrielRoiter de um dado $\Lambda$-módulo indecomponível $M$ e a de seus submódulos.

Lema 2.2.6. Seja $M$ um $\Lambda$-módulo indecomponível não simples. Então existe um submódulo $N$ tal que

$$
\mu(M)=\mu(N) \cup\{\ell(M)\}
$$

Demonstração. Como $M$ é indecomponível, pela definição de medida da Gabriel-Roiter, existe uma cadeia de submódulos indecomponíveis de $M, M_{1} \subsetneq M_{2} \subsetneq \ldots \subsetneq M_{n} \subsetneq M$ tal que $\mu(M)=\left\{\ell\left(M_{1}\right), \ldots, \ell\left(M_{n}\right), \ell(M)\right\}=\left\{\ell\left(M_{1}\right), \ldots, \ell\left(M_{n}\right)\right\} \cup\{\ell(M)\}$.

Seja $N_{1} \subsetneq N_{2} \subsetneq \ldots \subsetneq N_{s} \subsetneq M_{n}$ uma cadeia de submódulos indecomponíveis de $M_{n}$ tal que $\mu\left(M_{n}\right)=\left\{\ell\left(N_{1}\right), \ldots, \ell\left(N_{s}\right), \ell\left(M_{n}\right)\right\}$.

Como $M_{1} \subsetneq M_{2} \subsetneq \ldots \subsetneq M_{n}$ é uma cadeia de submódulos indecomponíveis de $M_{n}$, temos: $\left\{\ell\left(M_{1}\right), \ldots, \ell\left(M_{n}\right)\right\} \leq \mu\left(M_{n}\right)$.

Como $M_{n}$ é submódulo de $M$ temos $\ell\left(M_{n}\right)<\ell(M)$ então,

$$
\mu(M)=\left\{\ell\left(M_{1}\right), \ldots, \ell\left(M_{n}\right)\right\} \cup\{\ell(M)\} \leq \mu\left(M_{n}\right) \cup\{\ell(M)\} \leq \mu(M)
$$

De onde segue que $\mu(M)=\mu\left(M_{n}\right) \cup\{\ell(M)\}$.

Portanto, existe $N$ um submódulo próprio de $M$ tal que $\mu(M)=\mu(N) \cup\{\ell(M)\}$. 
Lema 2.2.7. Sejam $M$ um $\Lambda$-módulo indecomponível e $N \subset M$ um submódulo próprio indecomponível. São equivalentes:

1. $\mu(M)=\mu(N) \cup\{\ell(M)\}$;

2. todo submódulo próprio de $M$ tem medida no máximo $\mu(N)$.

Demonstração. (1) $\Rightarrow(2)$. Suponha que exista um submódulo próprio $N^{\prime}$ de $M$ tal que $\mu(N)<\mu\left(N^{\prime}\right)$. Então existe $a \in \mathbb{N}^{*}$, tal que a $\in \mu\left(N^{\prime}\right) \backslash \mu(N)$ e $[1, a-1]_{\mathbb{N}^{*}} \cap \mu\left(N^{\prime}\right)=[1, a-1]_{\mathbb{N}^{*}} \cap \mu(N)$. Como $N^{\prime}$ e $N$ são submódulos próprios de $M$, temos que $\ell(M)>\ell\left(N^{\prime}\right)$ e $\ell(M)>\ell(N)$. Observe que

$$
\left(\mu\left(N^{\prime}\right) \cup\{\ell(M)\}\right) \backslash(\mu(N) \cup\{\ell(M)\})=\mu\left(N^{\prime}\right) \backslash \mu(N)
$$

e

$$
[1, a-1]_{\mathbb{N}^{*}} \cap\left(\mu\left(N^{\prime}\right) \cup\{\ell(M)\}\right)=[1, a-1]_{\mathbb{N}^{*}} \cap(\mu(N) \cup\{\ell(M)\}),
$$

isto é, $\mu(M)=\mu(N) \cup\{\ell(M)\}<\mu\left(N^{\prime}\right) \cup\{\ell(M)\}$, absurdo.

$(2) \Rightarrow(1)$. Seja $N^{\prime}$ um submódulo próprio de $M$ tal que $\mu(M)=\mu\left(N^{\prime}\right) \cup\{\ell(M)\}$ (existe pelo Lema 2.2.6). Como $N^{\prime}$ é submódulo próprio de $M$, então por (2) temos que $\mu\left(N^{\prime}\right) \leq \mu(N)$. Temos ainda que $\ell(N)<\ell(M)$ e $\ell\left(N^{\prime}\right)<\ell(M)$, pois $N^{\prime}$ e $N$ são submódulos próprios de $M$. Então $\mu(M)=\mu\left(N^{\prime}\right) \cup\{\ell(M)\} \leq \mu(N) \cup\{\ell(M)\} \leq \mu(M)$ (a última desigualdade segue da definição da medida de Gabriel-Roiter do $\Lambda$-módulo $M$ ). Logo $\mu(M)=\mu\left(N^{\prime}\right) \cup\{\ell(M)\}=\mu(N) \cup\{\ell(M)\}$.

Definição 2.2.8. Uma inclusão $N \subsetneq M$ de $\Lambda$-módulos indecomponíveis é chamada de inclusão de Gabriel-Roiter se satisfizer uma das condições do lema acima.

Observemos que, se $N \subset M$ é inclusão de Gabriel-Roiter, então não existe submódulo $N^{\prime} \subseteq M$ indecomponível tal que $N \subsetneq N^{\prime} \subsetneq M$. No entanto, a recíproca desse resultado não é verdadeira, como nos mostra o exemplo a seguir.

Exemplo 2.2.9. No Exemplo 2.2.4, temos a inclusão $P_{b} \subset M_{1}$, que é uma inclusão de Gabriel-Roiter, pois

$$
\mu\left(M_{1}\right)=\mu\left(P_{b}\right) \cup\left\{\ell\left(M_{1}\right)\right\}=\{1,2\} \cup\{4\}=\{1,2,4\}
$$


Note que $P_{c}$ é um submódulo maximal indecomponível de $M_{1}$, mas $\mu\left(P_{c}\right)=\{1,3\}<\mu\left(P_{b}\right)=\{1,2\}$. Logo, $P_{c}$ não é uma inclusão de Gabriel-Roiter.

Para alguns módulos indecomponíveis, a medida de Gabriel-Roiter é facilmente calculada; é o caso, por exemplo, dos módulos indecomponíveis com socle simples.

Proposição 2.2.10. Seja $M$ um $\Lambda$-módulo indecomponível. São equivalentes:

(a) socM é simples.

(b) Para todo submódulo indecomponível $N$ de $M, \mu(N)=[1, \ell(N)]_{\mathbb{N}^{*}}$.

(c) $\mu(M)=[1, \ell(M)]_{\mathbb{N}^{*}}$.

Demonstração. $(a) \Rightarrow(b)$ Vamos mostrar por indução em $n=\ell(N)$, onde $N$ é um submódulo indecomponível de $M$. Para $n=1, N$ é simples e portanto temos $\operatorname{soc}(N)=N$ e $\mu(N)=\{1\}$. Para $n>1$, seja $N^{\prime}$ um submódulo maximal de $N\left(N^{\prime} \subsetneq N\right)$. Como $\operatorname{soc}(N)$ é simples, temos $\operatorname{soc}\left(N^{\prime}\right)=\operatorname{soc}(N)$, logo $N^{\prime}$ é indecomponível (se $N^{\prime}$ não fosse indecomponível, então $N^{\prime}=N_{1} \oplus N_{2}$, com $N_{1} \neq 0, N_{2} \neq 0$. Portanto, existiriam submódulos simples $S_{1} \subset N_{1}$ e $S_{2} \subset N_{2}$. Logo, $S_{1} \oplus S_{2} \subset \operatorname{soc}\left(N^{\prime}\right)$. Contradição).

Como $N^{\prime} \subsetneq N$ é maximal, temos $\ell\left(N^{\prime}\right)=\ell(N)-1$; junto com a hipótese de indução temos

$$
\mu\left(N^{\prime}\right)=\left[1, \ell\left(N^{\prime}\right)\right]_{\mathbb{N}^{*}}=[1, \ell(N)-1]_{\mathbb{N}^{*}} .
$$

Logo, existe uma cadeia de sumódulos indecomponíveis $N_{1} \subset N_{2} \subset \ldots \subset N_{t}=N^{\prime}$, onde $i=\ell\left(N_{i}\right)$ e $t=\ell\left(N^{\prime}\right)=\ell(N)-1$. Então temos $N_{1} \subset N_{2} \subset \ldots \subset N^{\prime} \subset N$ uma cadeia de de submódulos indecomponíveis de $N$, onde $\left\{\ell\left(N_{1}\right), \ell\left(N_{2}\right), \ldots, \ell(N)\right\}=[1, \ell(N)]_{\mathbb{N}^{*}}$.

Portanto, $\mu(N)=[1, \ell(N)]_{\mathbb{N}^{*}}$.

$(b) \Rightarrow(c)$ Nada há a demonstrar.

$(c) \Rightarrow(a)$ Vamos mostrar por indução em $m=\ell(M)$. Se $m=1$, nada há a demonstrar. Suponha $m>1$ e que todo módulo indecomponível $N$ com $\ell(N)<m$ e $\mu(N)=[1, \ell(N)]_{\mathbb{N}^{*}}$ tem socle simples.

Por hipótese, existe uma seqüência

$$
0=M_{0} \subseteq M_{1} \subseteq \ldots \subseteq M_{m}=M
$$


onde $M_{i} \in$ ind $\Lambda$ para todo $i=1, \ldots, m$.

Logo, por hipótese de indução, $M_{1}, \ldots, M_{m-1}$ têm socle simples. Escreva, em particular, $\operatorname{soc}\left(M_{m-1}\right)=S_{1}$. Suponha agora que $M$ não tem socle simples e considere um simples $S_{2}$ tal que $S_{1} \oplus S_{2}$ seja somando de $\operatorname{soc}(M)$. Como $S_{2}$ não é somando do $\operatorname{soc}\left(M_{m-1}\right)$, temos $S_{2} \cap M_{m-1}=\{0\}$. Por outro lado, como $M_{m-1}$ é submódulo maximal de $M$, a soma direta $S_{2} \oplus M_{m-1}$ é igual a $M$, uma contradição, pois $M$ é indecomponível e o resultado está provado.

Corolário 2.2.11. Se I é um $\Lambda$-módulo injetivo indecomponível então $\mu(I)=[1, \ell(I)]_{\mathbb{N}^{*}}$.

Demonstração. Como $I$ é injetivo indecomponível, então $\operatorname{soc}(I)$ é simples e pela Proposição 2.2.10 temos que $\mu(I)=[1, \ell(I)]_{\mathbb{N}^{*}}$.

Vejamos alguns casos em que a medida de Gabriel-Roiter é facilmente calculada.

- $\mu(\{0\})=\emptyset$;

- Se $S$ é simples, então $\mu(S)=\{1\}$, pois o módulo simples não possui submódulos não triviais;

- Se $M$ é indecomponível de comprimento 2 , então $\mu(M)=\{1,2\}$. Note que, nesse caso, o socle é simples e pela Proposição 2.2.10 temos o resultado;

- Se $M$ é indecomponível de comprimento 3, com socle simples, então $\mu(M)=\{1,2,3\}$ segue pela Proposição 2.2.10;

- Se $M$ é indecomponível de comprimento 3 , com socle de comprimento 2 , então $\mu(M)=\{1,3\}$, pois o módulo possui estrutura semelhante ao $P_{c}$ do Exemplo 2.2.4;

- Se $M$ é indecomponível de comprimento 4 , com socle simples, temos $\mu(M)=\{1,2,3,4\}$ segue da Proposição 2.2.10; 
- Se $M$ é indecomponível de comprimento 4, com socle de comprimento 2, podemos ter: $\mu(M)=\{1,2,4\}$ ou $\mu(M)=\{1,3,4\}$. O primeiro caso acontece quando o módulo possuir uma estrutura semelhante ao módulo $M_{1}$ do Exemplo 2.2.4 ou se

tiver uma estrutura semelhante a

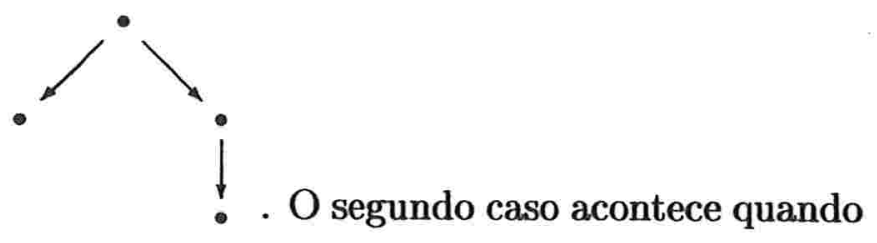

tiver uma estrutura semelhante a

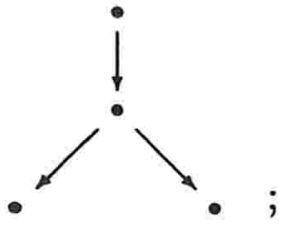

- Se $M$ é indecomponível de comprimento 4, com socle de comprimento 3 , temos $\mu(M)=\{1,4\}$, pois neste caso o módulo possui a estrutura

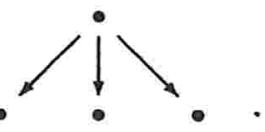

A medida de Gabriel-Roiter $\mu(M)$ de um $\Lambda$-módulo $M$ pode ser considerada como um número racional (entre 0 e 1) que depende apenas do comprimento dos submódulos indecomponíveis de $M$. Considere $\mathcal{P}_{b}\left(\mathbb{N}^{*}\right)=\left\{I \subset \mathbb{N}^{*}, I\right.$ é finito e $\left.1 \in I\right\}$. Notemos que $\mu(M)$ pertence a $\mathcal{P}_{b}\left(\mathbb{N}^{*}\right)$ (pois $M$ é finitamente gerado e $1 \in \mu(M)$ ). Existe uma aplicação injetora de $\mathcal{P}_{\boldsymbol{b}}\left(\mathbb{N}^{*}\right)$ no intervalo de números racionais $[0,1]$ como segue:

Proposição 2.2.12. A aplicação $r: \mathcal{P}_{b}\left(\mathbb{N}^{*}\right) \longrightarrow[0,1]$ dada por

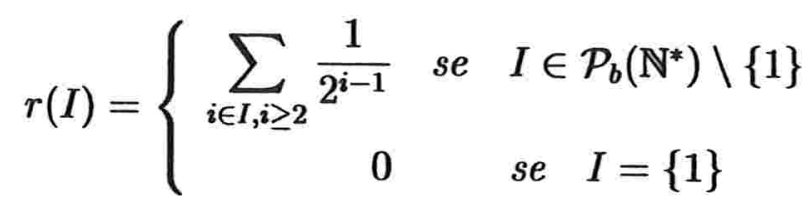

é injetiva e preserva a ordem.

Demonstração. É claro que a imagem de $r$ está contida no intervalo dos racionais de $[0,1]$. Para as outras afirmações, basta mostrar que se $I<J$, então $r(I)<r(J)$.

Sejam $I, J \in \mathcal{P}_{f}\left(\mathbb{N}^{*}\right)$ com $I<J$. Então $r(I)=r(I \cap J)+r(I \backslash J)$ e $r(J)=r(I \cap J)+r(J \backslash I) . \quad$ Seja $a$ o menor elemento de $J \backslash I . \quad$ Então 
$r(J \backslash I) \geq \frac{1}{2^{a-1}}=\sum_{i>a} \frac{1}{2^{i-1}}>r(I \backslash J)$, visto que $I \backslash J$ é um subconjunto próprio de $\left\{i \in \mathbb{N}^{*} \mid i>a\right\}$. Logo, $r(I)<r(J)$ e o resultado está provado.

Vamos aplicar $r$ em um conjunto de medidas de Gabriel-Roiter, conforme o exemplo a seguir:

Exemplo 2.2.13. No Exemplo 2.2.3, temos:

$\{1\}<\{1,4\}<\{1,3\}<\{1,3,5\}<\{1,2\}$.

Aplicando a função r em cada medida de Gabriel-Roiter temos:

$\frac{1}{2^{4-1}}=\frac{1}{8}<\frac{1}{2^{3-1}}=\frac{1}{4}<\frac{1}{2^{3-1}}+\frac{1}{2^{5-1}}=\frac{5}{16}<\frac{1}{2^{2-1}}=\frac{1}{2}$.

\subsection{Propriedade Principal de Gabriel}

Em seu trabalho [Ri], Ringel apresenta um teorema por ele chamado Propriedade Principal de Gabriel, visto que muitos resultados decorrem deste teorema, inclusive para demonstração do Teorema de Roiter. Resultados esses que nos mostram que basta conhecer apenas as medidas de Gabriel-Roiter dos módulos indecomponíveis, uma vez que todo módulo pode ser decomposto como soma direta de indecomponíveis. Além disso, o injetivo indecomponível de maior comprimento possui medida de Gabriel-Roiter maximal.

O teorema que vem a seguir é o teorema que Ringel chamou de Propriedade Principal de Gabriel.

Teorema 2.3.1. Sejam $X, Y_{1}, \ldots, Y_{n} \Lambda$-módulos indecomponíveis não nulos $e$ suponhamos que existe um monomorfismo $f: X \longrightarrow \oplus_{i=1}^{n} Y_{i}$.

(a) Então $\mu(X) \leq \max \mu\left(Y_{i}\right)$.

(b) Se $\mu(X)=\max \mu\left(Y_{i}\right)$, então $f$ cinde.

(c) Se max $\mu\left(Y_{i}\right)$ começa com $\mu(X)$, então existe algum $j$ tal que $\pi_{j} f$ é injetora, onde $\pi_{j}: \oplus_{i=1}^{n} Y_{i} \longrightarrow Y_{j}$ é a projeção canônica. 
Demonstração. Notemos que $(c) \Rightarrow(b)$. De fato, se $\mu(X)=\max \mu\left(Y_{i}\right)$, temos que $\max \mu\left(Y_{i}\right)$ começa com $\mu(X)$ e por $(c)$ sabemos que existe um índice $j$ tal que a composição $\pi_{j} f: X \longrightarrow \oplus_{i=1}^{n} Y_{i} \longrightarrow Y_{j}$ é injetora. Da injetividade de $\pi_{j} f: X \longrightarrow Y_{j}$ segue que $\mu(X) \leq \mu\left(Y_{j}\right)$. Desde que $\mu\left(Y_{j}\right) \leq \max \mu\left(Y_{i}\right)=\mu(X)$, temos $\mu\left(Y_{i}\right)=\mu(X)$, de onde segue que $\ell\left(Y_{j}\right)=\ell(X)$ (pois $X$ e $Y_{i}$ são indecomponíveis e pela Observação 2.2.2 item (c)).

Portanto, $\pi_{j} f: X \longrightarrow Y_{j}$ é bijetora, isto é, $f$ é um monomorfismo que cinde.

Faremos, a seguir, a demonstração simultânea de $(a)$ e $(c)$.

Seja $Y=\oplus_{i=1}^{n} Y_{i}$. Usaremos a indução sobre $t=\ell(X)+\ell(Y)$.

Como $X$ é não nulo, segue que $t \geq 2$.

Se $t=2$ e como $X$ é $\Lambda$-módulo indecomponível não nulo, segue que $\ell(X)=\ell(Y)=1$. Nesse caso temos que $X$ e $Y$ são simples. Então $\mu(X)=\mu(Y)=\{1\}$, (a) está provado, nesse caso.

O item (c) é imediato, visto que nesse caso, a projeção é o própria identidade.

Suponhamos $t>2$ e que o teorema é válido para monomorfismos $f: X \longrightarrow Y$, com $2 \leq \ell(X)+\ell(Y)<t$. Podemos ter dois casos:

(I) Existe $m \in\{1, \ldots, n\}$ tal que $\pi_{m} f$ não é sobrejetora.

Seja $Y_{i}^{\prime}=I m \pi_{i} f$, submódulo de $Y_{i}$. Decomponhamos $Y_{i}^{\prime}=\oplus_{j} Y_{i j}$, onde os $Y_{i j}$ são $\Lambda$-módulos indecomponíveis. Como $Y_{i j}$ são submódulos de $Y_{i}^{\prime}$ e $Y_{i}^{\prime}$ é submódulo de $Y_{i}$, então os $Y_{i j}$ são submódulos de $Y_{i}$.

Logo, $\mu\left(Y_{i j}\right) \leq \mu\left(Y_{i}\right)(*)$.

Notemos que $f^{\prime}: X \longrightarrow \oplus_{i=1}^{n} Y_{i}^{\prime}=\oplus_{i, j} Y_{i j}$ é monomorfismo ( $f^{\prime}$ foi obtido da $f$ restringindo o contradomínio para $\left.\oplus_{i=1}^{n} I m \pi_{i} f=\oplus_{i=1}^{n} Y_{i}^{\prime}\right)$ e como $\oplus_{i=1}^{n} Y_{i}^{\prime} \subset Y$ (pois $\pi_{m} f$ não é sobrejetora), temos

$$
\ell(Y)>\ell\left(\oplus_{i=1}^{n} Y_{i}^{\prime}\right)=\ell\left(\oplus_{i, j} Y_{i j}\right)=\sum_{i, j} \ell\left(Y_{i j}\right) .
$$

Logo, $t=\ell(X)+\ell(Y)>\ell(X)+\sum_{i, j} \ell\left(Y_{i j}\right)$.

Prova de $(a)$ : Por hipótese de indução $\mu(X) \leq \max _{i j}\left(Y_{i j}\right)$ e com $(*)$ temos 


$$
\mu(X) \leq \max _{i j}\left(Y_{i j}\right) \leq \max \mu\left(Y_{i}\right)(* *) .
$$

Portanto, $\mu(X) \leq \max \left(Y_{i}\right)$ e nesse caso $(a)$ está provado.

Prova de $(c)$ : Se $\max \mu\left(Y_{i}\right)$ começa com $\mu(X)$, e vale $(* *)$, então pelo Lema 2.1.3 (b) que $\max \mu\left(Y_{i j}\right)$ começa com $\mu(X)$ e, por hipótese de indução, existe uma projeção $\pi_{r s}: \oplus_{i j} Y_{i j} \longrightarrow Y_{r s}$ tal que a composição $f^{\prime}: X \longrightarrow \oplus_{i, j} Y_{i j}$, com $\pi_{r s}$ é um monomorfismo (isto é, $\pi_{r s} f^{\prime}$ é injetora).

Observemos que $\pi_{r s}=\pi_{s} \circ \pi_{r}^{\prime}\left(\pi_{r s}\right.$ é a composição da projeção $\pi_{r}^{\prime}: \oplus_{i j} Y_{i j}=\oplus_{i} Y_{i}^{\prime} \longrightarrow Y_{r}^{\prime}$ com a projeção $\left.\pi_{s}: Y_{r}^{\prime}=\oplus_{j} Y_{r j} \longrightarrow Y_{r s}\right)$. Como $\pi_{r s} f^{\prime}=\left(\pi_{s} \circ \pi_{r}^{\prime}\right) \circ f^{\prime}=\pi_{s} \circ\left(\pi_{r}{ }^{\prime} \circ f^{\prime}\right)$ é um monomorfismo, temos que $\pi_{r}{ }^{\prime} \circ f^{\prime}$ é monomorfismo. Se compusermos esse monomorfismo com o monomorfismo $Y_{r}^{\prime} \hookrightarrow Y_{r}$, obtemos $\pi_{r} f$ é injetora e nesse caso (c) está provado.

(II) Suponhamos que $\pi_{i} f$ é sobrejetora para todo $i$

Podemos subdividir esse caso em duas situações:

(II.1) Existe $j$ tal que $\pi_{j} f: X \longrightarrow Y_{j}$ é isomorfismo.

Como $X \cong Y_{j}$, segue que $\mu(X)=\mu\left(Y_{i}\right)$. E (a) está provado.

Como $\pi_{j} f$ é um isomorfismo, em particular é injetora. E (c) está provado.

(II.2) Para cada $i, \pi_{i} f$ não é isomorfismo.

Para $(a)$ : Como $\pi_{i} f: X \longrightarrow Y_{i}$ não é isomorfismo, em particular não é injetiva, temos

$$
\ell(X)>\ell\left(Y_{i}\right) \text {, para todo } i(* * *)
$$

Concluímos de $(* * *)$ que: $X$ não pode ser simples, pois neste caso $\ell(X)=1$ e $\ell\left(Y_{i}\right)=0$. Segue que, $f$ não seria injetora. Em particular, resulta que $\ell(X) \geq 2$ e pelo Lema 2.2.6, existe um submódulo indecomponível $X^{\prime}$ de $X$ tal que o monomorfismo $g: X^{\prime} \longrightarrow X$ é uma inclusão de Gabriel-Roiter, isto é, $X^{\prime} \hookrightarrow X$ tal que $\mu(X)=\mu\left(X^{\prime}\right) \cup\{\ell(X)\}$.

Notemos que $\ell\left(X^{\prime}\right)<\ell(X)$ e que $f g: X^{\prime} \longrightarrow \oplus Y_{i}$ é um monomorfismo, segue por hipótese de indução sobre $f g$ que $\mu\left(X^{\prime}\right) \leq \max \mu\left(Y_{i}\right)$.

O caso $\mu\left(X^{\prime}\right)=\max \mu\left(Y_{i}\right)$ pode ser desconsiderado pelo seguinte fato: Por (c) implica (b) a inclusão $X^{\prime} \hookrightarrow Y$ seria monomorfismo que cinde e esta aplicação se 
fatora através de $X^{\prime} \hookrightarrow X$, e nesse caso $g: X^{\prime} \hookrightarrow X$ seria monomorfismo que cinde. Porém, $X$ é indecomponível.

Portanto, $\mu\left(X^{\prime}\right) \leq \max \mu\left(Y_{i}\right)$ e consequentemente existe medida tal que $\mu\left(X^{\prime}\right)<\mu\left(Y_{j}\right)$. Logo, existe um número natural $a$ que pertence a $\mu\left(Y_{j}\right) \backslash \mu\left(X^{\prime}\right) \mathrm{e}$

$$
\mu\left(X^{\prime}\right) \cap[1, a-1]_{\mathbb{N}^{*}}=\mu\left(Y_{j}\right) \cap[1, a-1]_{\mathbb{N}^{*}}(* * * *)
$$

Como $a \notin \mu\left(X^{\prime}\right)$, segue que $a \neq \ell\left(X^{\prime}\right)$. De $a \in \mu\left(Y_{j}\right) \backslash \mu\left(X^{\prime}\right)$, temos que $a \leq \ell\left(Y_{j}\right)<\ell(X)$ (a segunda desigualdade decorre de $(* * *)$ ). Portanto, $a \in \mu\left(Y_{j}\right) \backslash \mu(X)$ e segue que $\mu(X) \cap[1, a-1]_{\mathbb{N}^{*}}=\mu\left(X^{\prime}\right) \cap[1, a-1]_{\mathbb{N}^{*}}=\mu\left(Y_{j}\right) \cap[1, a-1]_{\mathbb{N}^{*}}$ (observe que a segunda igualdade segue de $(* * * *))$ e nesse caso $(a)$ está provado.

Para $(c)$ : por $(* * *)$ temos $\ell(X)>\ell\left(Y_{j}\right)$ para todo $i$ e este caso exclui a possibilidade de max $\left(Y_{i}\right)$ começar com $\mu(X)$ (de fato, se existir algum $r$ tal que $\mu\left(Y_{r}\right)$ começa com $\mu(X)$, teremos $\ell(X) \leq \ell\left(Y_{r}\right)$. Contradição).

E assim está completa a demonstração de $(a)$ e de $(c)$.

Corolário 2.3.2. Se $M_{1}, \ldots, M_{t}$ são $\Lambda$-módulos indecomponíveis, então

$$
\mu\left(\oplus_{i=1}^{t} M_{i}\right)=\max \mu\left(M_{i}\right) .
$$

Demonstração. Como os $M_{i}$ 's são submódulos de $\oplus M_{i}$, temos que $\mu\left(M_{i}\right) \leq \mu\left(\oplus M_{i}\right)$, para todo $i \in\{1, \ldots, t\}$. Logo, $\max \mu\left(M_{i}\right) \leq \mu\left(\oplus M_{i}\right)$ (I).

Seja agora $M^{\prime}$ um submódulo indecomponível de $\oplus M_{i}$. Vamos mostrar que $\mu\left(M^{\prime}\right) \leq \max \mu\left(M_{i}\right)$.

Como existe um monomorfismo $\iota: M^{\prime} \hookrightarrow \oplus_{i} M_{i}$, por item (a) do Teorema 2.3.1 temos: $\mu\left(M^{\prime}\right) \leq \max \mu\left(M_{i}\right)$

Como $M^{\prime}$ é um submódulo indecomponível arbitrário, logo

$$
\mu\left(\oplus M_{i}\right) \leq \max \mu\left(M_{i}\right)(I I) .
$$

(pois $\mu\left(\oplus M_{i}\right)=\max \left\{\mu\left(M^{\prime}\right) \mid M^{\prime} \subset M, M^{\prime} \in i n d \Lambda\right\}$. O resultado segue então de $(I)$ e $(I I)$. 
Corolário 2.3.3. Se $M \subseteq N$ e $\mu(N) \leq \mu(M)$, então $M$ contém um somando direto indecomponivel de $N$.

Demonstração. Decomponhamos $N$ em soma direta de indecomponíveis, $N=N_{1} \oplus \ldots \oplus N_{r}$. Pelo Corolário 2.3.2, temos que $\mu(N)=\max \mu\left(N_{i}\right)$. Podemos ter duas possibilidades para $M$ :

(i) $M$ é indecomponível. Por hipótese, existe um monomorfismo $\iota: M \hookrightarrow N_{1} \oplus \ldots \oplus N_{r}$. Então pelo Teorema 2.3.1 item $(a)$, temos $\mu(M) \leq \max \mu\left(N_{i}\right)$. Por outro lado, como $\max \mu\left(N_{i}\right) \leq \mu(M)$, segue que $\mu(M)=\max \mu\left(N_{i}\right)$. Por item (b) do Teorema 2.3.1, o monomorfismo $\iota$ cinde e portanto $M$ é um somando direto de $N_{1} \oplus \ldots \oplus N_{r}$.

(ii) $M$ não é indecomponível. Seja $M=\oplus_{i=1}^{t} M_{i}$ em soma direta de indecomponíveis, pelo Corolário 2.3.2 segue que $\mu(M)=\max \mu\left(M_{i}\right)$. Seja $j$ o índice tal que $\mu\left(M_{j}\right)=\max \mu\left(M_{i}\right)$. Note que $M_{j} \subset M \subset N$ e $\max \mu\left(N_{i}\right)=\mu(N) \leq \mu\left(M_{j}\right)$, pelo caso (i) temos que $M_{j}$ é um somando direto indecomponível de $N$. Como $M_{j} \subset M$, segue que $M$ contém um somando direto indecomponível de $N$.

Corolário 2.3.4. Seja $M$ um $\Lambda$-módulo indecomponível. Então $M$ é um injetivo de maior comprimento se e somente se $\mu(M)$ é maximal.

Demonstração. $(\Rightarrow)$ Suponhamos que $M$ seja o $\Lambda$-módulo injetivo de maior comprimento. Seja $N$ um módulo indecomponível. Sabemos que existe o monomorfismo $N \hookrightarrow I_{0}(N)$, onde $I_{0}(N)$ é a envolvente injetiva de $N$ e $I_{0}(N)=I_{1} \oplus \ldots \oplus I_{r}$ pode ser decomposto como soma direta de injetivos indecomponíveis. Pelo Teorema 2.3.1 (a)

$$
\mu(N) \leq \max \mu\left(I_{i}\right)=\mu\left(I_{0}(N)\right)(*) .
$$

Temos $\mu\left(I_{0}(N)\right)=\mu\left(\oplus_{i=1}^{r} I_{i}\right)=\max \left\{\mu\left(I_{i}\right)\right\}=\max \left[1, \ell\left(I_{i}\right)\right]_{\mathbb{N}^{*}} \leq \mu(M)$. Dessa desigualdade junto com $(*)$, segue que $\mu(N) \leq \mu\left(I_{0}(N)\right) \leq \mu(M)$. Logo, $\mu(M)$ é maximal.

$(\Leftarrow)$ Suponha agora que $\mu(M)$ seja maximal. Pelo Lema 1.2.6, existe uma envolvente injetiva $\iota: M \hookrightarrow \oplus I_{i}$, onde $I_{i}$ são injetivos indecomponíveis. Como $\mu(M)$ é maximal, temos $\mu\left(I_{i}\right) \leq \mu(M)$ para todo $i$. Logo $\max \mu\left(I_{i}\right) \leq \mu(M)$, e pelo item $(a)$ do Teorema 2.3.1, temos $\mu(M) \leq \max \mu\left(I_{i}\right)$, de onde segue que $\mu(M)=\max \mu\left(I_{i}\right)$. Do item (b) do 
mesmo teorema segue que $\iota$ cinde, de onde temos que $M$ é um injetivo indecomponível de maior comprimento.

Observação 2.3.5. Observe que os extremos do intervalo das medidas de Gabriel-Roiter são $\{1\}$ e $[1, \ell(I)]_{\mathbb{N}^{*}}$, onde I é o injetivo indecomponível de maior comprimento.

$\left[1, \ell^{\circ}(I)\right]_{N^{*}}$




\section{Capítulo 3}

\section{Teorema de Roiter}

Em sua versão para a demonstração do Teorema de Roiter, Gabriel leva em consideração a existência de uma partição na categoria de ind $\Lambda$ (onde $\Lambda$ é uma álgebra de tipo de representação limitado), explorando a noção de injetivos relativos em subcategorias. Esse resultado de Gabriel e Roiter motivou Auslander e Smalø em [AS], a introduzirem a noção de partição pré-injetiva.

Em [AS], Auslander e Smalø descrevem condições suficientes sobre uma categoria $\mathcal{C}$ para a existência e unicidade da chamada partição pré-injetiva. Nosso interesse centra-se no caso $\mathcal{C}=i n d \Lambda$, visto que nosso objetivo é apresentar a demonstração do Teorema de Roiter proposta por Gabriel, utilizando conceitos e terminologias mais modernos apresentados em [AS], por Auslander e Smalø como: cocobertura de categorias, subcategorias contravariantemente finitas e a medida de Gabriel-Roiter (estudada no capítulo anterior). Esses conceitos, e outros resultados que serão necessários para o entendimento da demonstração do Teorema de Roiter, serão apresentados neste capítulo.

\subsection{Cocoberturas}

No estudo de uma subcategoria $\mathcal{C}$ de $\bmod \Lambda$, às vezes, é conveniente também considerar as subcategorias $a d d \mathcal{C}$ e $i n d \mathcal{C}$, que iremos descrever agora.

Seja $\mathcal{C}$ uma subcategoria de $\bmod \Lambda$. Denotamos por $a d d \mathcal{C}$ a subcategoria de $\bmod \Lambda$ 
formada por todos $\Lambda$-módulos cujos somandos diretos indecomponíveis são somandos de elementos de $\mathcal{C}$. A categoria indC é a subcategoria de $\mathcal{C}$ formada por todos os módulos indecomponíveis de $\mathcal{C}$.

Definição 3.1.1. Sejam $\mathcal{C}$ uma subcategoria de mod $\Lambda$ e $\mathcal{B}$ uma subcategoria de ind $\Lambda$, tais que $\mathcal{B} \subset \mathcal{C}$. Diz-se que $\mathcal{B}$ é uma cocobertura de $\mathcal{C}$ se para qualquer $X \in \mathcal{C}$ existir um monomorfismo $f: X \longrightarrow M^{\prime}$, com $M^{\prime} \in$ addB. $E$ uma cocobertura $\mathcal{B}$ de $\mathcal{C}$ é uma cocobertura minimal de $\mathcal{C}$ quando nenhuma subcategoria própria de $\mathcal{B}$ for uma cocobertura de $\mathcal{C}$.

Definição 3.1.2. Seja $\mathcal{C}$ uma subcategoria de $\bmod \Lambda$. Diz-se que um $\Lambda$-módulo $M \in \mathcal{C}$ é um $\mathcal{C}$-injetivo se todo monomorfismo $f: M \longrightarrow Y$, com $Y \in$ addC, cinde.

Denotaremos por $\mathcal{I}_{0}(\mathcal{C})$ a subcategoria de indC formada pelos $\mathcal{C}$-injetivos indecomponiveis.

\section{Observação 3.1.3.}

(i) Um módulo $M$ é um mod $\Lambda$-injetivo se e somente se $M$ for injetivo.

De fato, seja $M$ injetivo. Consideremos o monomorfismo $f: M \longrightarrow Y$, com $Y \in \bmod \Lambda$. Como $M$ é injetivo, para o morfismo id $: M \longrightarrow M$, existe $h: Y \longrightarrow M$ tal

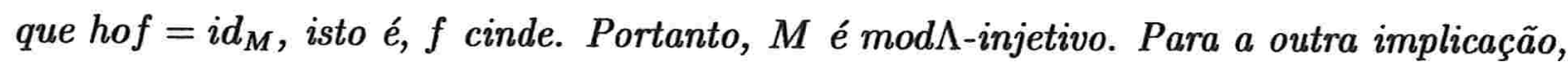
seja $M$ um $\mathcal{C}$-injetivo e considere o monomorfismo $\iota: M \hookrightarrow I_{0}(M)$, onde $I_{0}(M)$ é a envolvente injetiva de $M$. Como $M$ é um $\mathcal{C}$-injetivo, ८ cinde. Logo $M$ é um somando direto de injetivos.

(ii) O conjunto formado pelos injetivos indecomponíveis é uma cocobertura minimal de $\bmod \Lambda$. De fato, basta lembrar que todo $M \in \bmod \Lambda$ possui uma envolvente injetiva $M \hookrightarrow I_{0}(M)$, onde $I_{0}(M)$ pode ser decomposta como soma direta de injetivos indecomponiveis.

Exemplo 3.1.4. Consideremos a álgebra $\Lambda$ do Exemplo 2.2.4 e as subcategorias de mod $\Lambda$ $\mathcal{C}=\left\{P_{a}, P_{b}, P_{c}, S_{b}, M_{1}, M_{2}\right\}$ e $\mathcal{B}=\left\{M_{1}, M_{2}\right\}$. Então, $\mathcal{B}$ é uma cocobertura de $\mathcal{C}$, pois existem os monomorfismos $P_{a} \hookrightarrow M_{1}, P_{b} \hookrightarrow M_{1}, P_{c} \hookrightarrow M_{1}, S_{b} \hookrightarrow M_{1}, M_{1} \hookrightarrow M_{1} e$ $M_{2} \hookrightarrow M_{2}$. Como não existe monomorfismo de $M_{2}$ para cópias de $M_{1}$ e nem de $M_{1}$ para cópias de $M_{2}$, segue que $\mathcal{B}$ é uma cocobertura minimal de $\mathcal{C}$. 
Notemos, ainda, que $M_{1}, M_{2}$ são $\mathcal{C}$-injetivos, pois o monomorfismo $f: M_{1} \longrightarrow X$, com $X \in \mathcal{C}$ só é possível quando $X=M_{1}$ e, além disso, ele cinde. De forma análoga, o monomorfismo $g: M_{2} \longrightarrow Y$, com $Y \in$ addC só é possível quando $Y=M_{2}$ e cinde.

$$
\text { Logo, } \mathcal{I}_{0}(\mathcal{C})=\left\{M_{1}, M_{2}\right\}
$$

Observação 3.1.5. Observemos que o conceito de $\mathcal{C}$-injetivo generaliza, de certa maneira, o conceito de injetivo para uma subcategoria $\mathcal{C}$. No entanto, nem todas as propriedades dos injetivos se generalizam. Por exemplo, não vale a propriedade de levantamento para $\mathcal{C}$-injetivos, isto é, se $f: M \longrightarrow N$ é um monomorfismo em $\mathcal{C}$ e $\phi: M \longrightarrow Y$ é um morfismo, com $Y \in \mathcal{I}_{0}(\mathcal{C})$, nem sempre existe $g: N \longrightarrow Y$ tal que gof $=\phi$. No exemplo acima, tomemos o monomorfismo $P_{c} \hookrightarrow M_{1}$ e a projeção $P_{c} \longrightarrow M_{2}$, com $M_{2}$ um $\mathcal{C}$-injetivo, mas não existe morfismo não nulo $M_{1} \longrightarrow M_{2}$. Logo, não vale a propriedade do levantamento.

Proposição 3.1.6. Seja $\mathcal{C}$ uma subcategoria de mod $\Lambda$. Se $\mathcal{B}$ é uma cocobertura de $\mathcal{C}$, então $\mathcal{I}_{0}(\mathcal{C}) \subseteq \mathcal{B}$

Demonstração. Seja $I \in \mathcal{I}_{0}(\mathcal{C})$. Como $\mathcal{B}$ é uma cocobertura de $\mathcal{C}$, então existe um monomorfismo $f: I \longrightarrow M^{\prime}$, com $M^{\prime} \in$ addB.

Como $I$ é um $\mathcal{C}$-injetivo, então o monomorfismo $f$ cinde. De onde segue que $I$ é isomorfo a um somando indecomponível de $M^{\prime}$. Logo, $I \in \mathcal{B}$. Portanto, $\mathcal{I}_{0}(\mathcal{C}) \subseteq \mathcal{B}$.

Para a demonstração do próximo teorema, necessitamos da seguinte proposição cuja demostração se encontra-se em [AS].

Proposição 3.1.7. Sejam $M$ e $N \in \bmod \Lambda$ com $M$ indecomponivel. Suponhamos que $f_{i}: M \longrightarrow M, i=1,2, \ldots, n$ seja uma familia finita de morfismos $e f_{0}: M \longrightarrow N$ um morfismo tais que o morfismo induzido $f: M \longrightarrow M^{n} \oplus N$ seja um monomorfismo que não cinde. Então existe um monomorfismo $M \longrightarrow N^{m}$, onde $N^{m}$ é uma soma finita de cópias de $N$.

Teorema 3.1.8. Sejam $\mathcal{C}$ e $\mathcal{B}$ subcategorias de ind $\Lambda$ tais que $\mathcal{B} \subset \mathcal{C}$. Então $\mathcal{B}$ é uma cocobertura minimal de $\mathcal{C}$ se e só se $\mathcal{B}=\mathcal{I}_{0}(\mathcal{C})$. 
Demonstração. Pela Proposição 3.1.6, temos que, se $\mathcal{B}$ é uma cocobertura de $\mathcal{C}$, então $\mathcal{I}_{0}(\mathcal{C}) \subseteq \mathcal{B}$. Logo, se $\mathcal{B}=\mathcal{I}_{0}(\mathcal{C})$ é cocobertura de $\mathcal{C}$, então $\mathcal{B}$ é uma cocobertura minimal de $\mathcal{C}$.

Resta mostrarmos que, se $\mathcal{B}$ é cocobertura minimal de $\mathcal{C}$, então $\mathcal{B}=\mathcal{I}_{0}(\mathcal{C})$. Suponhamos que $\mathcal{B}$ é uma cocobertura de $\mathcal{C}$ e que $\mathcal{B} \supsetneq \mathcal{I}_{o}(\mathcal{C})$, isto é, existe $M \in \mathcal{B} \backslash \mathcal{I}_{0}(\mathcal{C})$. Como $M \notin \mathcal{I}_{0}(\mathcal{C})$, então existe um monomorfismo $g: M \longrightarrow Y$, com $Y \in \operatorname{addC}$, que não cinde.

Por outro lado, temos que, de $\mathcal{B}$ ser cocobertura de $\mathcal{C}$, existe um monomorfismo $h: Y \longrightarrow X, \operatorname{com} X \in a d d \mathcal{B}$.

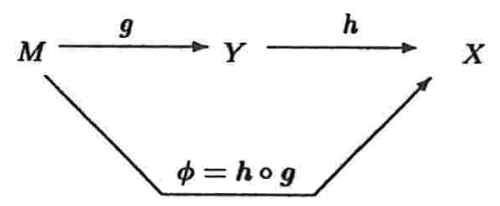

Como $g$ não cinde, segue que a composta $\phi=h \circ g: M \longrightarrow X$ é um monomorfismo que não cinde. Logo, podemos escrever $X=M^{n} \oplus N$, onde $N$ não tem somando direto de $M$. Segue da Proposição 3.1.7, que existe um monomorfismo $M \longrightarrow N^{m}$. Como $N \in \operatorname{add}(\mathcal{B} \backslash\{M\})$, temos que $\mathcal{B} \backslash\{M\}$ é uma cocobertura para $\mathcal{C}$. Logo, se existir $M \in \mathcal{B} \backslash \mathcal{I}_{0}(\mathcal{C})$, então $\mathcal{B}$ é uma cocobertura não minimal de $\mathcal{C}$. Portanto, de $\mathcal{B}$ ser cocobertura minimal de $\mathcal{C}$ implica que $\mathcal{B}=\mathcal{I}_{0}(\mathcal{C})$.

Como conseqüência imediata desse teorema, temos:

Corolário 3.1.9. Seja $\mathcal{C}$ uma subcategoria de ind $\Lambda$.

(a) Se $\mathcal{B}_{1}, \mathcal{B}_{2}$ são cocoberturas minimais de $\mathcal{C}$, então $\mathcal{B}_{1}=\mathcal{B}_{2}=\mathcal{I}_{0}(\mathcal{C})$.

(b) Se $\mathcal{C}$ tem cocobertura finita, então $\mathcal{I}_{0}(\mathcal{C})$ é uma cocobertura minimal finita.

Demonstração. (a) Sejam $\mathcal{B}_{1}$ e $\mathcal{B}_{2}$ duas cocoberturas minimais para $\mathcal{C}$. Então, pelo Teorema 3.1.8, temos $\mathcal{B}_{1}=\mathcal{I}_{0}(\mathcal{C})=\mathcal{B}_{2}$. 
(b) Se $\mathcal{B}$ é uma cocobertura finita de $\mathcal{C}$, então existe uma subcategoria $\mathcal{B}^{\prime}$ de $\mathcal{B}$ com o menor número de objetos não isomorfos tais que $\mathcal{B}^{\prime}$ é cocobertura de $\mathcal{C}$. Pelo Teorema 3.1.8, $\mathcal{B}^{\prime}=\mathcal{I}_{0}(\mathcal{C})$, o que mostra que $\mathcal{I}_{0}(\mathcal{C})$ é uma cocobertura minimal finita para $\mathcal{C}$.

\subsection{Subcategorias contravariantemente finitas}

Nesta seção, estudaremos o conceito de subcategoria contravariantemente finita introduzido por Auslander e Smalø em [AS].

Definição 3.2.1. Sejam $\mathcal{C}$ e $\mathcal{D}$ subcategorias de $\bmod \Lambda$ tais que $\mathcal{C} \subset \mathcal{D}$. Dizemos que $\mathcal{C}$ é contravariantemente finita em $\mathcal{D}$ se para todo $M \in$ addD , existe um morfismo $f_{M}: N \longrightarrow$ $M$ com $N \in$ addC tal que $\left(X, f_{M}\right): \operatorname{Hom}_{\Lambda}(X, N) \longrightarrow \operatorname{Hom}_{\Lambda}(X, M)$ onde $h \longmapsto g=f \circ h$, é sobrejetora para qualquer $X \in$ addC. Nesse caso, o morfismo $f_{M}$ é chamado de uma $\mathcal{C}$-aproximação à esquerda de $M$. Ou seja o diagrama abaixo é comutativo.

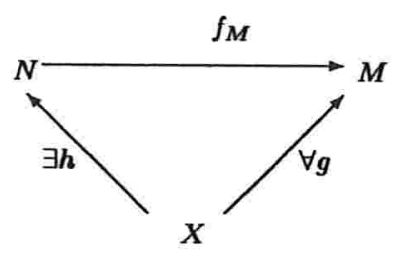

Dizemos que $\mathcal{C}$ é contravariantemente finita quando $\mathcal{C}$ é contravariantemente finita em $\bmod \Lambda$.

\section{Observação 3.2.2.}

Se $M \in \mathcal{C}$, a identidade id $: M \longrightarrow M$ é uma $\mathcal{C}$-aproximaçãa à esquerda de $M$, pois $(X, i d): \operatorname{Hom}_{\Lambda}(X, M) \rightarrow \operatorname{Hom}_{\Lambda}(X, M)$ é sobrejetora para todo $X \in$ addC.

Se Hom $(\mathcal{C}, M)=0$ (isto é, para todo $X \in$ addC o único morfismo $f: X \longrightarrow M$ possivel é o morfismo nulo), então qualquer morfismo nulo que tem como contradomínio $M$ é uma $\mathcal{C}$-aproximação de $M$.

Veremos agora exemplos de subcategorias contravariantemente finitas. 
Exemplo 3.2.3. Um exemplo trivial de subcategoria $\mathcal{C}$ contravariantemente finita ocorre quando $\mathcal{C}=i n d \Lambda$, pois para cada $M \in$ ind $\Lambda$, basta considerarmos $f=i d: M \longrightarrow M e$, $(X, i d): \operatorname{Hom}_{\Lambda}(X, M) \longrightarrow \operatorname{Hom}_{\Lambda}(X, M)$ é sobrejetora, para todo $X \in$ addC.

Exemplo 3.2.4. Considerando a álgebra $\Lambda$ do Exemplo 2.2.4 e $\mathcal{C}=\left\{M_{1}, P_{a}, P_{b}, P_{c}, S_{b}\right\}$ uma subcategoria de ind $\Lambda$, vamos mostrar que $\mathcal{C}$ é contravariantemente finita.

Se $X \in \mathcal{C}$, pelo exemplo anterior nada temos a demonstrar. Então vamos considerar os casos em que $X \notin \mathcal{C}$.

Se $X=I_{a}$, um morfismo não nulo $\varphi: M \longrightarrow X$ é possível quando $M=P_{a}, P_{b}, P_{c}$ ou $M_{1}$. Nesse caso, consideremos a $\mathcal{C}$-aproximação à esquerda de $I_{a}$ um morfismo irredutível $M_{1} \longrightarrow I_{a}$.

Se $X=I_{b}$, um morfismo não nulo $\varphi: M \longrightarrow X$ é possível quando $M=S_{b}, P_{b}, P_{c}$ ou $M_{1}$. Nesse caso, consideremos a $\mathcal{C}$-aproximação à esquerda de $I_{b}$ um morfismo irredutivel $M_{1} \longrightarrow I_{b}$.

Se $X=I_{c}$, um morfismo não nulo $\varphi: M \longrightarrow X$ é possível quando $M=P_{c}$ ou $M_{1}$. Nesse caso, consideremos a $\mathcal{C}$-aproximação à esquerda de $I_{c}$ a composta dos morfismos irredutiveis $M_{1} \longrightarrow I_{b}$ com $I_{b} \longrightarrow I_{c}$.

Se $X=M_{2}$, o morfismo não nulo $\varphi: M \longrightarrow X$ é possível quando $M=P_{a}, P_{b}, P_{c}$ ou $M_{1}$. Nesse caso, consideremos a $\mathcal{C}$-aproximação à esquerda de $M_{2}$ o morfismo irredutível $P_{c} \longrightarrow M_{2}$.

Portanto, $\mathcal{C}$ é contravariantemente finita.

Para a demonstração do Teorema de Roiter necessitaremos de alguns fato como do fato que toda subcategoria cofinita (lembramos que $\mathcal{C}$ é cofinita em ind $\Lambda$ se existir um número finito de módulos indecomponíveis que não pertencem a $\mathcal{C}$ ) é contravariantemente finita. Comecemos com a seguinte proposição:

Proposição 3.2.5. Se $\mathcal{C}$ for contravariantemente finita, então $\mathcal{C}$ tem uma cocobertura finita.

Demonstração. Consideremos $I_{1}, \ldots, I_{n}$ cópias de todos os injetivos indecomponíveis dois a dois isomorfos. Para cada $j$, consideremos $f_{j}: M_{j} \longrightarrow I_{j}$ a $\mathcal{C}$-aproximação à esquerda 
de $I_{j}$. Seja $\mathcal{B}=\left\{M_{1}, M_{2}, \ldots, M_{n}\right\}$. Para provar que $\mathcal{B}$ é uma cocobertura, precisamos mostrar que todo módulo em $\mathcal{C}$ pode ser imerso em cópias de módulos de $\mathcal{B}$.

Seja $X \in \mathcal{C}$ e consideremos sua envolvente injetiva $\iota: X \rightarrow I_{0}(X)$. Como $I_{0}(X)=I_{1}^{r_{1}} \oplus \ldots \oplus I_{n}^{r_{n}}$, consideremos o morfismo

$$
f=\left(f_{1}^{r_{1}}, \ldots, f_{n}^{r_{n}}\right): M_{1}^{r_{1}} \oplus \ldots \oplus M_{n}^{r_{n}} \longrightarrow I_{0}(X)
$$

Agora,

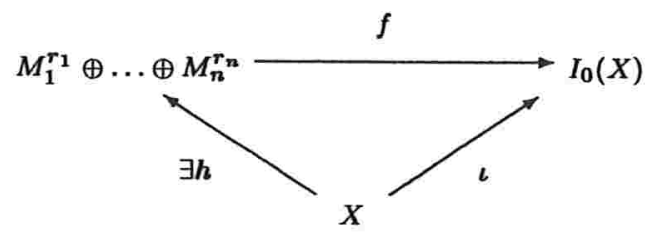

Pela construção de $f$, existe $h: X \longrightarrow M_{1}^{r_{1}} \oplus \ldots \oplus M_{n}^{r_{n}}=M^{\prime}$ tal que $f \circ h=\iota$.

Como $\iota$ é um monomorfismo, $h$ também o será, logo existe um monomorfismo $X \hookrightarrow M^{\prime}$ $\operatorname{com} M^{\prime} \in \operatorname{add}(\mathcal{B})$.

Portanto, $\mathcal{B}$ é uma cocobertura de $\mathcal{C}$.

Veremos agora exemplos de cocoberturas de algumas subcategorias.

Exemplo 3.2.6. Consideremos a álgebra do Exemplo 2.2.5: a $k$-álgebra $\Lambda=k \Delta$, onde $\Delta$ é o quiver:

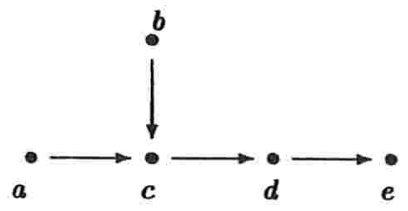

Sejam $\mathcal{I}_{0}=\{$ injetivos indecomponiveis $\}, \mathcal{C}_{1}=i n d \Lambda \backslash \mathcal{I}_{0}, \mathcal{I}_{1}=\left\{\mathcal{C}_{1}\right.$-injetivos indecomponíveis $\}$ e por indução $\mathcal{C}_{i}=i n d \Lambda \backslash\left(\mathcal{I}_{0} \cup \ldots \cup \mathcal{I}_{i-1}\right)$ e $\mathcal{I}_{i}=\left\{\mathcal{C}_{i}\right.$-injetivos indecomponíveis $\}$, para $i=1,2, \ldots$.

Na demonstração da Proposição 3.2.5 vimos que para acharmos uma cocobertura finita dos $\mathcal{C}_{i}$, basta encontrarmos as $\mathcal{C}_{i^{-}}$aproximação à esquerda, $f: M_{i} \longrightarrow I_{i}$, dos injetivos 
indecomponíveis $I_{1}, I_{2}, \ldots, I_{n}$, pois nesse caso, $\left\{M_{i}\right\}_{i=1}^{t}$ é uma cocobertura finita de $\mathcal{C}_{i}$. Vejamos novamente o quiver de Auslander-Reiten:

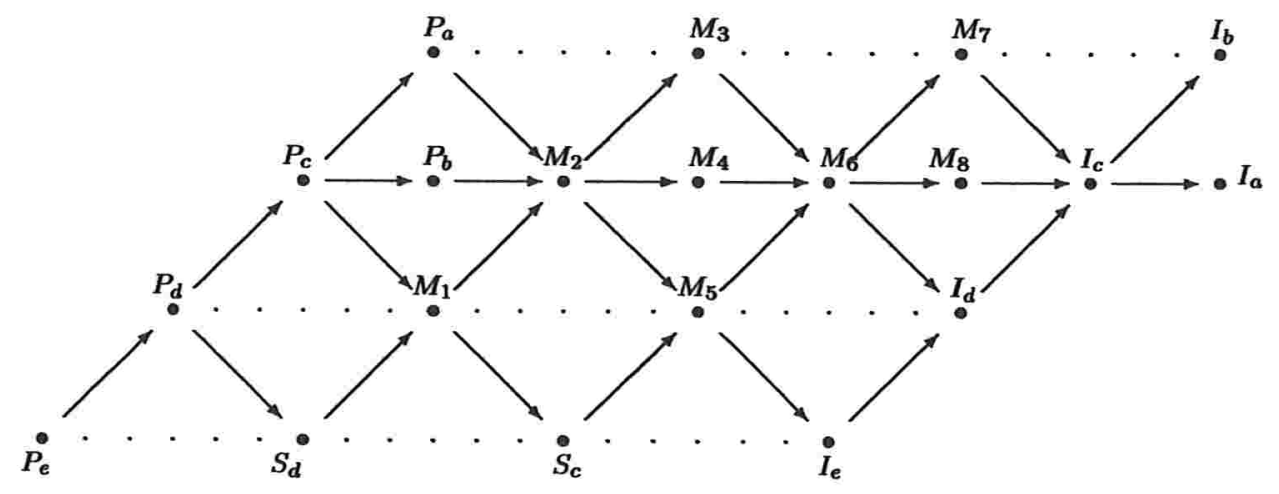

$A \mathcal{C}_{i}$-aproximação à esquerda de cada injetivo indecomponível ou é um morfismo irredutivel ou uma soma de compostas de morfismos irredutíveis. De fato,

Para $\mathcal{C}_{1}$, temos que a $\mathcal{C}_{1}$-aproximação à esquerda de:

- $I_{a}$ é o morfismo $M_{7} \longrightarrow I_{a}$;

- $I_{b}$ é o morfismo $M_{8} \longrightarrow I_{b}$;

- $I_{c}$ é o morfismo $M_{8} \oplus M_{7} \longrightarrow I_{c}$;

- $I_{d}$ é o morfismo $M_{6} \longrightarrow I_{d}$;

- $I_{e}$ é o morfismo $M_{5} \longrightarrow I_{e}$.

Portanto, uma cocobertura finita para $\mathcal{C}_{1}$ é $\left\{M_{5}, M_{6}, M_{8} \oplus M_{7}, M_{7}\right\}$, e a cocobertura minimal é $\left\{M_{5}, M_{6}, M_{7}, M_{8}\right\}=\mathcal{I}_{1}$.

Para $\mathcal{C}_{2}$, temos que a $\mathcal{C}_{2}$-aproximação à esquerda de:

- $I_{a}$ é o morfismo $M_{4} \longrightarrow I_{a}$;

- $I_{b}$ é o morfismo $M_{3} \longrightarrow I_{b}$;

- $I_{c}$ é o morfismo $S_{c} \oplus M_{3} \oplus M_{4} \longrightarrow I_{c}$; 
- $I_{d}$ é o morfismo $M_{4} \oplus M_{3} \longrightarrow I_{d}$;

- $I_{e}$ é o morfismo $M_{2} \longrightarrow I_{e}$.

Portanto, uma cocobertura finita para $\mathcal{C}_{2}$ é $\left\{M_{4}, M_{3}, S_{c} \oplus M_{3} \oplus M_{4}, M_{4}\right\}$, e a cocobertura minimal é $\left\{S_{c}, M_{2}, M_{3}, M_{4}\right\}=\mathcal{I}_{2}$.

Para $\mathcal{C}_{3}$, temos que a $\mathcal{C}_{3}$-aproximação à esquerda de:

- $I_{a}$ é o morfismo $P_{a} \longrightarrow I_{a}$;

- $I_{b}$ é o morfismo $P_{b} \longrightarrow I_{b}$;

- $I_{c}$ é o morfismo $M_{1} \oplus P_{a} \oplus P_{b} \longrightarrow I_{c}$;

- $I_{d}$ é o morfismo $M_{1} \oplus P_{a} \oplus P_{b} \longrightarrow I_{d}$;

- $I_{e}$ é o morfismo $P_{a} \oplus P_{b} \longrightarrow I_{e}$.

Portanto, uma cocobertura finita para $\mathcal{C}_{3}$ é $\left\{P_{a}, P_{b}, M_{1} \oplus P_{a} \oplus P_{b}, P_{a} \oplus P_{b}\right\}$ e a cocobertura minimal é $\left\{P_{a}, P_{b}, M_{1}\right\}=\mathcal{I}_{3}$.

$\operatorname{Para~}_{4}:$

- Se $X=I_{a}, I_{b}$, temos $\operatorname{Hom}_{\Lambda}\left(\mathcal{C}_{4}, X\right)=0$, logo qualquer morfismo nulo $M \longrightarrow X$, para $\forall M \in \mathcal{C}_{4}$ é uma $\mathcal{C}_{4}$-aproximação à esquerda de $X$.

- $O$ morfismo $P_{c} \longrightarrow I_{c}$ é a $\mathcal{C}_{4}$-aproximação à esquerda de $I_{c}$;

- $O$ morfismo $S_{d} \oplus P_{c} \longrightarrow I_{d}$ é a $C_{4}$ - aproximaçãa à esquerda de $I_{d}$;

- $O$ morfismo $P_{c} \longrightarrow I_{e}$ é a $\mathcal{C}_{4}$-aproximação à esquerda de $I_{e}$.

Portanto, uma cocobertura finita para $\mathcal{C}_{4}$ é $\left\{P_{c}, S_{d} \oplus P_{c}\right\}$ e a cocobertura minimal é $\left\{P_{c}, S_{d}\right\}=\mathcal{I}_{4}$.

Para $\mathcal{C}_{5}$ :

- $S e X=I_{a}, I_{b}, I_{c}$, temos $\operatorname{Hom}_{\Lambda}\left(\mathcal{C}_{5}, X\right)=0$, logo qualquer morfismo nulo $M \longrightarrow X$, com $M \in \mathcal{C}_{5}$ é uma $\mathcal{C}_{5}$-aproximação à esquerda de $X$. 
- $O$ morfismo $P_{d} \longrightarrow I_{d}$ é a $\mathcal{C}_{5}$-aproximação à esquerda de $I_{d}$;

- $O$ morfismo $P_{d} \longrightarrow I_{e}$ é a $\mathcal{C}_{5}$ aproximação à esquerda de $I_{e}$.

Portanto, uma cocobertura finita para $\mathcal{C}_{5}$ é $\left\{P_{d}\right\}$ que é também a cocobertura minimal, $\mathcal{I}_{5}=\left\{P_{d}\right\}$

Para $\mathcal{C}_{6}$ :

- Se $X=I_{a}, I_{b}, I_{c}, I_{d}$, temos $\operatorname{Hom}_{\Lambda}\left(\mathcal{C}_{6}, X\right)=0$, logo qualquer morfismo nulo desse conjunto é uma $\mathcal{C}_{6}$-aproximação à esquerda de $X$;

- $A \mathcal{C}_{6}$-aproximação à esquerda de $I_{e}$ é o morfismo $P_{e} \longrightarrow I_{e}$.

Portanto, a cocobertura minimal para $\mathcal{C}_{6}$ é $\left\{P_{e}\right\}=\mathcal{I}_{6}$.

Notemos que a propriedade de ter-se morfismos quase cindidos à direita não é "herdada"diretamente pela subcategoria. Contudo se a subcategoria for finita, temos o seguinte lema:

Lema 3.2.7. Sejam $\mathcal{C} \subset \mathcal{D}$ subcategorias de mod $\Lambda$. Se $\mathcal{D}$ tem morfismos quase cindidos $\grave{a}$ direita $e \mathcal{C}$ é contravariantemente finita em $\mathcal{D}$, então $\mathcal{C}$ tem morfismos quase cindidos $\grave{a}$ direita.

Demonstração. Seja $N$ indecomponível em $\mathcal{C}$. Como $\mathcal{C} \subset \mathcal{D}$, segue que $N \in$ indD . Como $\mathcal{D}$ tem morfismos quase cindidos à direita, então existe um morfismo quase cindido à direita $f: M \longrightarrow N$, com $M \in a d d \mathcal{D}$, isto é, existe $M \in$ addD e $(X, f): \operatorname{Hom}_{\Lambda}(X, M) \longrightarrow \operatorname{Hom}_{\Lambda}(X, N)$ tem imagem $\operatorname{rad}(X, N)$ para todo $X \in \mathcal{D}$.

Mas $\mathcal{C}$ é contravariantemente finita em $\mathcal{D}$, então existe $f_{M}: M_{\mathcal{C}} \longrightarrow M$, com $M_{\mathcal{C}} \in$ addC tal que $\operatorname{Im}\left(X, f_{M}\right)=\operatorname{Hom}_{\Lambda}(X, M)$ para todo $X \in \operatorname{addC}$, onde $\left(X, f_{M}\right)$ é o morfismo de $\operatorname{Hom}_{\Lambda}\left(X, M_{\mathcal{C}}\right)$ em $\operatorname{Hom}_{\Lambda}(X, M)$ dado por $h \longmapsto f_{M}$ o $h$.

Assim, a composta $g=f \circ f_{M}: M_{\mathcal{D}} \longrightarrow N$ é tal que $\operatorname{Im}(X, g)=\operatorname{rad}(X, N)$ para todo $X \in \mathcal{C}$. Logo, $\mathcal{C}$ tem morfismos quase cindidos à direita.

$\mathrm{Na}$ seqüência, veremos alguns resultados que serão importantes para a demonstração do Teorema de Roiter. 
Proposição 3.2.8. Se $\mathcal{C}$ é uma subcategoria cofinita em ind $\Lambda$, então $\mathcal{C}$ é contravariantemente finita em ind $\Lambda$.

Demonstração. Como $\mathcal{C}$ é cofinita, então chamemos $\mathcal{A}$ a subcategoria finita de ind $\Lambda$ tal que ind $\Lambda \backslash \mathcal{A}=\mathcal{C}$.

A prova será feita por indução sobre o número $n$ de elementos de $\mathcal{A}$.

Se $n=0$, então não há nada a demonstrar, pois ind $\backslash \mathcal{A}=$ ind $\Lambda$ que é contrariantemente finita (veja o Exemplo 3.2.3).

Seja $\mathcal{A}=\left\{N_{1}, \ldots, N_{n-1}, N_{n}\right\}$. Suponhamos que a hipótese de indução seja válida para todo os $\mathcal{A}^{\prime}$ com número de elementos menor do que $n$, isto é, tal que ind $\backslash \mathcal{A}^{\prime}$ é contravariantemente finita. Mostraremos que, para todo $N \in$ ind $\Lambda$, existe um morfismo $f: Y \longrightarrow N$, com $Y \in$ addC tal que para todo $X \in$ addC, $\operatorname{Im}\left((X, f): \operatorname{Hom}_{\Lambda}\left(X, M_{n}\right) \longrightarrow \operatorname{Hom}_{\Lambda}(X, N)\right)=\operatorname{Hom}_{\Lambda}(X, N)$, isto é, o diagrama abaixo é comutativo.

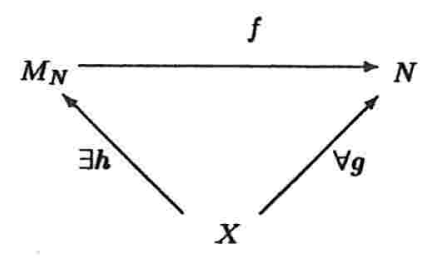

Seja $N \in$ ind $\Lambda$. Se $N \in \mathcal{C}$, então tomamos $f=i d$ (ver Observação 3.2.1).

Se $N \notin \mathcal{C}$, isto é, $N \in \mathcal{A}(=i n d \Lambda \backslash \mathcal{C})$ e sem perda de generalidade, podemos supor $N=N_{n}$.

Seja $\mathcal{A}^{\prime}=\left\{N_{1}, \ldots, N_{n-1}\right\}$. Pela hipótese de indução, ind $\backslash \backslash \mathcal{A}^{\prime}$ é contravariantemente finita. Então existe um morfismo $f: M \longrightarrow N_{n} \operatorname{com} M \in \operatorname{add}\left(\right.$ ind $\left.\Lambda \backslash \mathcal{A}^{\prime}\right)$ tal que

$$
\operatorname{Im}\left((X, f): \operatorname{Hom}_{\Lambda}(X, M) \rightarrow \operatorname{Hom}_{\Lambda}\left(X, N_{n}\right)\right)=\operatorname{Hom}_{\Lambda}\left(X, N_{n}\right),
$$

para todo $X \in \operatorname{add}\left(\operatorname{ind} \Lambda \backslash \mathcal{A}^{\prime}\right)$.

Se $N=N_{n}$ não é somando direto de $M$, então $M \in \operatorname{add}(i n d \Lambda \backslash \mathcal{A})$ e $f: M \longrightarrow N_{n}$ é tal que, para todo $X \in \operatorname{add}(\operatorname{ind} \Lambda \backslash \mathcal{A})$, temos $\operatorname{Im}(X, f)=\operatorname{Hom}_{\Lambda}(X, N)$.

Se $N=N_{n}$ é um somando direto de $M$, então podemos escrever $M=N_{n}^{l} \oplus M^{\prime}$, com $M^{\prime} \in \operatorname{add}(i n d \Lambda \backslash \mathcal{A})$. Sabemos que ind $\Lambda$ tem morfismos quase cindidos e, pela hipótese 
de indução, que (ind $\Lambda \backslash \mathcal{A}^{\prime}$ ) é contravariantemente finita, então pelo Lema 3.2.7, segue que ind $\Lambda \backslash \mathcal{A}^{\prime}$ tem morfismos quase cindidos à direita.

Seja $h: Y \longrightarrow N_{n}$ um morfismo quase cindido minimal à direita, $Y \in \operatorname{addind} \Lambda \backslash \mathcal{A}^{\prime}$. $\mathrm{E}$, nesse caso, $N_{n}$ não é somando direto de $Y$. Isto é, $Y \in \operatorname{add}(i n d \Lambda \backslash \mathcal{A})$. Então para todo $X \in \operatorname{add}(i n d \Lambda \backslash \mathcal{A})$, temos que $\operatorname{Im}\left((X, f): \operatorname{Hom}_{\Lambda}(X, Y) \longrightarrow \operatorname{Hom}_{\Lambda}\left(X, N_{n}\right)\right)=$ $\operatorname{rad}(X, N)$.

Olhemos para a composta $f \circ\left(h^{l}, i d_{M^{\prime}}\right): Y^{l} \oplus M^{\prime} \stackrel{\left(h^{l}, i d_{M^{\prime}}\right)}{\longrightarrow} N_{n}^{l} \oplus M^{\prime} \stackrel{f}{\longrightarrow} N=N_{n}$. Seja um morfismo $\sigma: X \longrightarrow N$, com $X \in \operatorname{add}($ ind $\Lambda \backslash \mathcal{A})$. Como ind $\Lambda \backslash \mathcal{A}^{\prime}$ é contravariantemente finita, existe $\left(\delta_{1}, \ldots, \delta_{l}, \delta\right)^{t}: X \longrightarrow N_{n}^{l} \oplus M^{\prime}$ tal que $f \circ\left(\delta_{1}, \ldots, \delta_{l}, \delta\right)^{t}=\sigma$.

Como $h$ é um morfismo quase cindido à direita e para cada $i=1, \ldots, l$, o morfismo $\delta_{i}: X \longrightarrow N_{n}$ não é epimorfismo que cinde (pois $N_{n}$ não é somando de $X$ ), então existe $p_{i}^{\prime}: X \longrightarrow Y$, tais que $\delta_{i}=h \circ p_{i}^{\prime}$. Seja $g=\left(p_{1}^{\prime}, p_{2}^{\prime}, \ldots, p_{t}^{\prime}, \delta\right)^{t}: X \longrightarrow Y^{l} \oplus M^{\prime}$ que é tal que $f \circ\left(h, i d_{M}^{\prime}\right)=\delta$. Vejamos o diagrama comutativo abaixo:

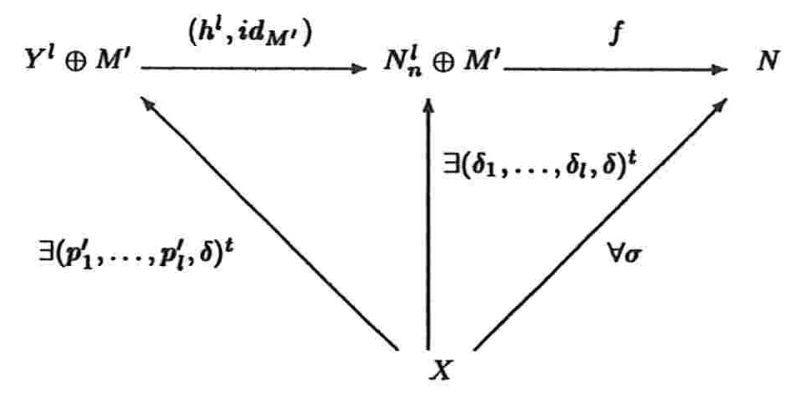

$\operatorname{Logo}, \operatorname{Im}\left(X, f \circ\left(h^{l}, i d M^{\prime}\right)\right)=\operatorname{Hom}_{\Lambda}(X, N)$ para todo $X \in \operatorname{add}(i n d \Lambda \backslash \mathcal{A}) \mathrm{e}$ $Y^{l} \oplus M^{\prime} \in \operatorname{add}(i n d \Lambda \backslash \mathcal{A})$. Portanto, como $\mathcal{A}$ é finita, temos que ind $\Lambda \backslash \mathcal{A}$ é contravariantemente finita, isto é, $\mathcal{C}$ é contravariantemente finita em ind $\Lambda$.

\subsection{Teorema de Roiter}

Nessa seção apresentaremos a demonstração do Teorema de Roiter proposta por Gabriel em [Ga]. Iniciaremos com dois lemas que nos serão úteis para esta prova. 
Lema 3.3.1. Se $\mathcal{C}$ é uma subcategoria cofinita em ind $\Lambda$, então existe apenas um número finito de $\mathcal{C}$-injetivos indecomponíveis.

Demonstração. Suponhamos que $\mathcal{C}$ seja uma subcategoria cofinita em ind $\Lambda$. Segue então pela Proposição 3.2.8, que $\mathcal{C}$ é uma subcategoria contravariantemente finita. Pela Proposição 3.2.5, temos que $\mathcal{C}$ tem uma cocobertura finita e por item $(b)$ do Corolário 3.1.8, existe uma cocobertura minimal finita que é a formada por todos os $\mathcal{C}$-injetivos indecomponíveis. Em particular, temos que existe apenas um número finito de $\mathcal{C}$-injetivos indecomponíveis.

Lema 3.3.2. Seja $\mathcal{C}$ uma subcategoria de ind $\Lambda$ e $N \in \mathcal{C}$ tal que $\mu(X) \leq \mu(N)$, para todo $X \in \mathcal{C}$. Então $N$ é um $\mathcal{C}$-injetivo.

Demonstração. Consideremos um monomorfismo $f: N \longrightarrow \oplus_{i=1}^{n} X_{i}$, com $X_{i} \in$ indC. Pelo item (a) do Teorema 2.3.1, sabemos que $\mu(N) \leq \max \mu\left(X_{i}\right)$. Como por hipótese $\mu(X) \leq \mu(N)$, para todo $X \in \mathcal{C}$, segue que $\mu(N)=\max \mu\left(X_{i}\right)$. E, pelo item (b) do mesmo teorema, temos que $f$ cinde, isto é, $N$ é um $\mathcal{C}$-injetivo.

Vejamos agora a demonstração do Teorema de Roiter.

Teorema 3.3.3. (Teorema de Roiter) Se existir um número natural não nulo $m$ tal $q u e \ell(M) \leq m$ para todo $\Lambda$-módulo indecomponível $M$, então $\Lambda$ é de tipo de representação finito.

Demonstração. Como por hipótese $\ell(M) \leq m$ para todo $M \in$ ind $\Lambda$, temos que $\mu(M) \subset[1, m]_{\mathbb{N}^{*}}$. Assim, existe apenas um número finito de possibilidades para a medida de Gabriel-Roiter dos $\Lambda$-módulos indecomponíveis. Sejam $\mu_{1}, \ldots, \mu_{t}$, com $\mu_{i}<\mu_{i+1}$, para $i=1, \ldots, t-1$, todas as possíveis medidas de Gabriel-Roiter dos $\Lambda$-módulos indecomponíveis. Sabemos, pelo Corolário 2.3.4, que $\mu(M)$ é maximal se e só se $M$ é um injetivo indecomponível de maior comprimento. Logo, $\mu_{t}=\mu(I)$, onde $I$ é um injetivo indecomponível de maior comprimento.

Seja $\mathcal{I}_{0}=$ injetivos indecomponíveis $\}$, que é finita (pois, existem apenas um número finito de injetivos indecomponíveis). $\mathrm{E} \mathcal{A}_{0}=\left\{X \in i n d \Lambda \mid \mu(X)=\mu_{t}\right\} \subset \mathcal{I}_{0}$ é finita. 
Seja $\mathcal{C}_{1}=\{M \in$ ind $\Lambda: M$ não contém submódulos injetivos $\}=i n d \Lambda \backslash \mathcal{I}_{0}$.

Se $\mathcal{C}_{1}=\emptyset$, então ind $\Lambda=\mathcal{I}_{0}$ é categoria finita.

Se $\mathcal{C}_{1} \neq \emptyset$, então existe $N_{1} \in \mathcal{C}_{1}$ tal que $\mu(X) \leq \mu\left(N_{1}\right)$, para todo $X \in \mathcal{C}_{1}$ e $1 \leq \mu\left(N_{1}\right) \leq \mu_{t-1}$. Além disso, pelo Lema 3.3.2, $N_{1}$ é um $\mathcal{C}_{1}$-injetivo.

Por outro lado, $\mathcal{C}_{1}$ é uma subcategoria cofinita em ind $\Lambda$ e portanto, pelo Lema 3.3.1, $\mathcal{C}_{1}$ admite apenas um número finito de $\mathcal{C}_{1}$-injetivos indecomponíveis; ou seja $\mathcal{I}_{1}=\left\{\mathcal{C}_{1}\right.$-injetivos indecomponíveis $\}$ é finita.

Observemos que $\mathcal{A}_{1}=\left\{X \in \operatorname{ind} \Lambda \mid \mu(X)=\mu_{t-1}\right\} \subset\left(\mathcal{I}_{0} \cup \mathcal{I}_{1}\right)$ (pois, se $X \in \mathcal{C}_{1}$ e $\mu(X)=\mu_{t-1}$ então, pelo Lema 3.3.2, $X \in \mathcal{I}_{1}$.)

Seja $\mathcal{C}_{2}=i n d \Lambda \backslash\left(\mathcal{I}_{0} \cup \mathcal{I}_{1}\right)$.

Se $\mathcal{C}_{2}=\emptyset$, então ind $\Lambda=\mathcal{I}_{0} \cup \mathcal{I}_{1}$ é finita

Se $\mathcal{C}_{2} \neq \emptyset$, por um processo indutivo, semelhante ao realizado acima, obtemos $j \in \mathbb{N}^{*}$, com $1 \leq j \leq t-1$ para qual a subcategoria $\mathcal{C}_{j}=i n d \Lambda \backslash\left(\mathcal{I}_{0} \cup \mathcal{I}_{1} \cup \ldots \cup \mathcal{I}_{j-1}\right) \neq \emptyset$, $\mathcal{I}_{j}=\left\{\mathcal{C}_{j}\right.$-injetivos indecomponíveis $\}$ é finita e $\mathcal{A}_{j}=\left\{X \in i n d \Lambda \mid \mu(X)=\mu_{t-j} \subset \bigcup_{m=0}^{j} \mathcal{I}_{m}\right.$, tal que $\mathcal{C}_{j+1}=i n d \Lambda \backslash\left(\mathcal{I}_{0} \cup \ldots \cup \mathcal{I}_{j}\right)=\emptyset$ (pois $\mathcal{A}_{t-1}=\{X \in \operatorname{ind} \Lambda \mid \mu(X)=1\}$ ).

Portanto, ind $\Lambda$ é uma categoria finita e o resultado está provado.

Notemos que, no Exemplo 3.2.6, fizemos uma partição de ind $\Lambda$ de tal modo que cada conjunto $\mathcal{I}_{i}$ tem um número finito de elementos e $i n d \Lambda=\bigcup_{i=0}^{6} \mathcal{I}_{i}$.

\subsection{Partição pré-injetiva}

A noção de subcategorias contravariantemente finitas foi introduzida por Auslander e Smalø em [AS] para a construção da chamada partição pré-injetiva. Mencionamos, da introdução desse trabalho, o seguinte trecho:

"O ímpeto original deste trabalho ... originou-se do esforço de explicar um antigo resultado de Gabriel e Roiter a respeito de álgebras de Artin de tipo de representação 
finito em termos de técnicas e idéias desenvolvidas por Auslander e Reiten com relação a seqüências quase cindidas e morfismos irredutíveis... "

Vamos, nesta seção, relembrar essa construção.

Uma coleção de subconjuntos $\mathcal{I}_{0}, \ldots, \mathcal{I}_{m}, \ldots, \mathcal{I}_{\infty}$ de $i n d \Lambda$ que cumpre:

1. $i n d \Lambda=\bigcup_{j \in \mathbb{N} \cup\{\infty\}} \mathcal{I}_{j}$

2. $\mathcal{I}_{i} \cap \mathcal{I}_{j}=\emptyset$ se $i \neq j$

3. Para todo $j<\infty, \mathcal{I}_{j}$ é uma cocobertura minimal finita de $i n d \Lambda \backslash \bigcup_{i<j} \mathcal{I}_{i}$ será chamada de partição pré-injetiva.

Vejamos agora quando que ind $\Lambda$ terá uma partição pré-injetiva.

Sejam $\mathcal{C}=i n d \Lambda$ e $\mathcal{X}$ uma subcategoria de $\mathcal{C}$. Denotamos por $\mathcal{C}_{\mathcal{X}}$ a subcategoria $\mathcal{C} \backslash \mathcal{X}$.

Já definimos $\mathcal{I}_{0}(\mathcal{C})$ como sendo a subcategoria de indC formada por $\mathcal{C}$-injetivos. Definimos $\mathcal{I}_{1}(\mathcal{C})=\mathcal{I}_{0}\left(\mathcal{C}_{\mathcal{I}_{0}(\mathcal{C})}\right)=\mathcal{I}_{0}\left(\mathcal{C} \backslash \mathcal{I}_{0}(\mathcal{C})\right)=\left\{\mathcal{C} \backslash \mathcal{I}_{0}(\mathcal{C})\right.$-injetivos $\}, \mathcal{I}_{2}(\mathcal{C})=\mathcal{I}_{0}\left(\mathcal{C}_{\mathcal{I}_{0} \cup \mathcal{I}_{1}}\right)$ e por indução $\mathcal{I}_{k}(\mathcal{C})=\mathcal{I}_{0}\left(\mathcal{C}_{\mathcal{I}_{0}(\mathcal{C}) \cup \ldots \cup \mathcal{I}_{k-1}(\mathcal{C})}\right), k \in \mathbb{N}^{*}$.

Essas subcategorias de $\mathcal{C}$ verifica as seguintes propriedades:

1. $\mathcal{I}_{i}(\mathcal{C}) \cap \mathcal{I}_{j}(\mathcal{C})=\emptyset$ para $i, j \leq \infty$ com $i \neq j$.

De fato, vamos supor $i<j$. Sabemos que $\mathcal{I}_{j}(\mathcal{C})=\mathcal{I}_{0}\left(\mathcal{C}_{\mathcal{I}_{0}(\mathcal{C}) \cup \ldots \cup \mathcal{I}_{i} \cup \ldots \cup \mathcal{I}_{j-1}(\mathcal{C})}\right)$. Logo, qualquer $M \in \mathcal{I}_{i}(\mathcal{C}) \subset \mathcal{I}_{0}(\mathcal{C}) \cup \ldots \cup \mathcal{I}_{i}(\mathcal{C}) \cup \ldots \cup \mathcal{I}_{j-1}(\mathcal{C})$, de onde segue que $M \notin \mathcal{I}_{j}(\mathcal{C})$. Portanto, $\mathcal{I}_{i} \cap \mathcal{I}_{j}=\emptyset$ se $i \neq j$

2. Se $\mathcal{I}_{i}(\mathcal{C})=\emptyset$, então $\mathcal{I}_{j}(\mathcal{C})=\emptyset$ para $i \leq j<\infty$.

De fato, vamos supor que $\mathcal{I}_{i}(\mathcal{C})=\emptyset$ e provaremos que $\mathcal{I}_{i+1}(\mathcal{C})=\emptyset$. Observemos que $\mathcal{I}_{i+1}(\mathcal{C})=\mathcal{I}_{0}\left(\mathcal{C} \backslash\left(\mathcal{I}_{0}(\mathcal{C}) \cup \ldots \cup \mathcal{I}_{i-1}(\mathcal{C}) \cup \mathcal{I}_{i}(\mathcal{C})\right)\right)=\mathcal{I}_{0}\left(\mathcal{C} \backslash\left(\mathcal{I}_{0}(\mathcal{C}) \cup \ldots \cup \mathcal{I}_{i-1}(\mathcal{C})\right)\right)=$ $\mathcal{I}_{i}(\mathcal{C})=\emptyset$. A segunda igualdade decorre da hipótese que $\mathcal{I}_{i}(\mathcal{C})=\emptyset$. Portanto, $\mathcal{I}_{i+1}(\mathcal{C})=\emptyset$.

Assim, a coleção $\left\{\mathcal{I}_{i}(\mathcal{C})\right\}_{i=0, \ldots, \infty}$ será uma partição pré-injetiva de $\mathcal{C}$ se $\mathcal{I}_{i}(\mathcal{C})$ for uma

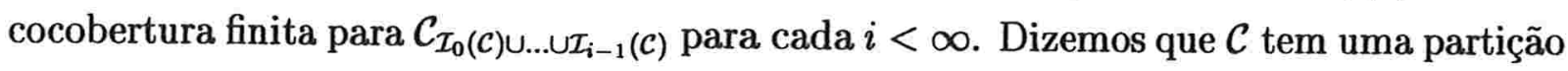
pré-injetiva se $\left\{\mathcal{I}_{i}(\mathcal{C})\right\}_{i=0, \ldots, \infty}$ é uma partição pré-injetiva de $\mathcal{C}$. 
Estamos agora em condições de apresentar uma idéia da demonstração do teorema da existência e unicidade de partição pré-injetiva. Para maiores detalhes, indicamos [AS].

Teorema 3.4.1. Seja $\Lambda$ uma álgebra de Artin. Então existe e é unica a partição pré-injetiva de ind $\Lambda$.

Demonstração. (Idéia) Sabemos que:

(i) A subcategoria $\mathcal{I}_{0}$ formada pelos representantes $\Lambda$-módulos injetivos indecomponíveis é a cocobertura minimal de $i n d \Lambda$.

(ii) (Lema 3.3.1) Se $\mathcal{C}$ for uma subcategoria cofinita em ind $\Lambda$, então existe um número finito de $\mathcal{C}$-injetivos indecomponíveis, isto é, $\mathcal{I}_{0}(\mathcal{C})$ é finita.

(iii) $\mathcal{I}_{0}(\mathcal{C})$ é a cocobertura minimal de $\mathcal{C}$.

Temos primeiramente que ind $\Lambda$ tem cocobertura minimal finita que é $\mathcal{I}_{0}$ (por (i)).

Se $i n d \Lambda=\mathcal{I}_{0}$, então está feito.

Se $\mathcal{C}_{1}=i n d \Lambda \backslash \mathcal{I}_{0} \neq \emptyset$, então $\mathcal{C}_{1}$ é cofinita em ind $\Lambda$. Logo $\mathcal{I}_{1}=\mathcal{I}_{0}\left(\mathcal{C}_{1}\right)$ é finita e é a cocobertura minimal de $\mathcal{C}_{1}$.

Considere $\mathcal{I}_{0}, \mathcal{I}_{1}$. Claramente $\mathcal{I}_{0} \cap \mathcal{I}_{1}=\emptyset$, pois $\mathcal{I}_{0} \subset \mathcal{C}_{1}$.

Se $i$ ind $\Lambda=\mathcal{I}_{0} \cup \mathcal{I}_{1}$, está feito.

Caso contrário, indutivamente podemos supor que tenhamos construído uma partição $\mathcal{I}_{0}, \mathcal{I}_{1}, \ldots, \mathcal{I}_{n-1}, \mathcal{I}$, com as seguintes propriedades:

1. $\bigcup_{i=0}^{n-1} \mathcal{I}_{i} \cup \mathcal{I}=i n d \Lambda$

2. $\mathcal{I}_{i} \cap \mathcal{I}_{j}=\emptyset$ se $i \neq j$ e $\mathcal{I} \cap \mathcal{I}_{i}=\emptyset i=0, \ldots, n-1$

3. Para cada $i \leq n-1, \mathcal{I}_{i}$ é a cocobertura minimal finita de $\bigcup_{j=i}^{n-1} \mathcal{I}_{j} \cup \mathcal{I}$.

Temos que construir $\mathcal{I}_{n}$. Como $\mathcal{I}$ é cofinita, então por (ii) temos que $\mathcal{I}_{0}(\mathcal{I})$ é finita. Segue por (iii) que $\mathcal{I}_{0}(\mathcal{I})$ é a cocobertura minimal de $\mathcal{I}$. Escolhemos $\mathcal{I}_{n}=\mathcal{I}_{0}(\mathcal{I})$ que é finita. 
Repetindo esse processo, encontramos a partição pré-injetiva.

Notemos que, na demonstração do Teorema de Roiter, construímos uma partição pré-injetiva de ind $\Lambda$ e, pelo Teorema 3.4.1, sabemos que essa partição pré-injetiva é unica. Logo, quando temos uma álgebra de tipo representação finito, existe uma partição pré-injetiva finita $\left\{\mathcal{I}_{i}\right\}_{i=0}^{t}$ de $i n d \Lambda$, para algum $t$.

Exemplo 3.4.2. Consideremos a $k$-álgebra $\Lambda=k \Delta$, onde $\Delta$ é o quiver:

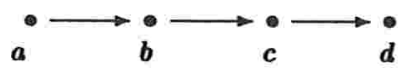

O quiver de Auslander-Reiten, $\Gamma_{\Lambda}$, é dado por:

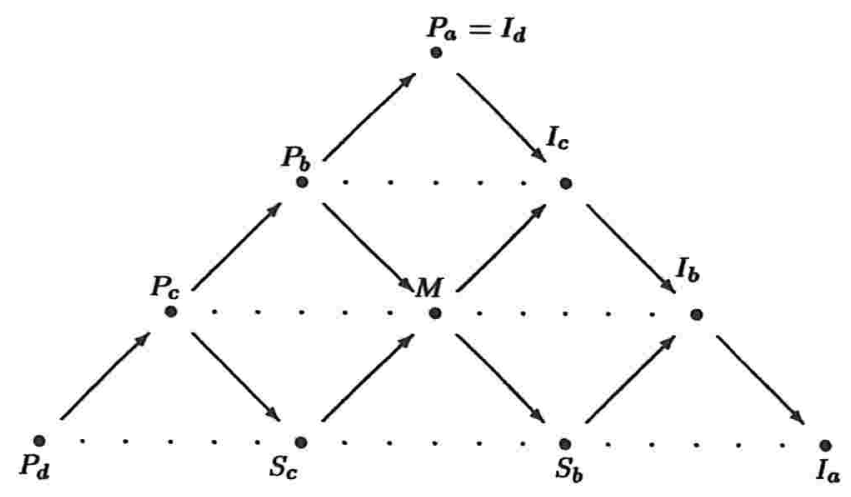

Representamos os módulos indecomponíveis como sendo:

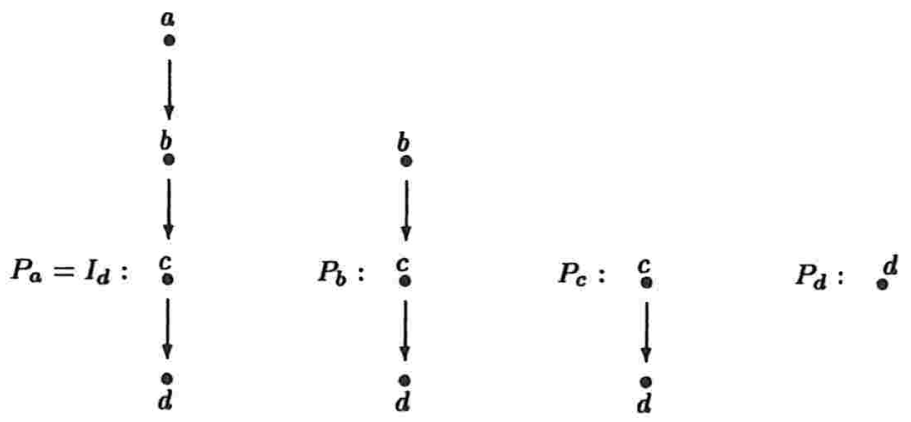




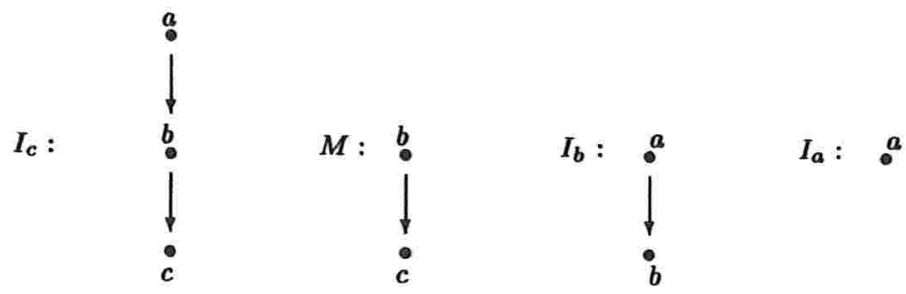

Vamos achar a partição pré-injetiva procedendo da mesma forma que na demonstração do Teorema de Roiter, isto é, calculando os $\mathcal{I}_{i}=\left\{\mathcal{C}_{i}\right.$-injetivos indecomponiveis $\}$, onde $\mathcal{C}_{i}=\operatorname{ind} \Lambda \backslash\left(\mathcal{I}_{0} \cup \mathcal{I}_{1} \cup \ldots \cup \mathcal{I}_{i-1}\right)$.

Considere $\mathcal{C}_{1}=\operatorname{ind} \Lambda \backslash \mathcal{I}_{0}$.

Para os $\Lambda$-módulos indecomponíveis $N=S_{b}, M$ e $P_{b}$, um monomorfismo $f: N \longrightarrow X$ em $\mathcal{C}_{1}$ só é possível para $X=N^{n}$, e esse morfismo cinde. Logo, $S_{b}, M$ e $P_{b}$ são $\mathcal{C}_{1}$ injetivos. mos que existem monomorfismos irredutiveis $S_{c} \longrightarrow M$ e $P_{c} \longrightarrow P_{b}$ que não cindem. Portanto, $\mathcal{I}_{1}=\left\{S_{b}, M, P_{b}\right\}$.

Considere $\mathcal{C}_{2}=i n d \Lambda \backslash\left(\mathcal{I}_{0} \cup \mathcal{I}_{1}\right)$.

Para os $\Lambda$-módulos indecomponiveis $N=P_{c}$ e $S_{c}$, um monomorfismo $N \longrightarrow X$, só é possivel para $X=N^{n}$, que cinde. Logo, $P_{c}$ e $S_{c}$ são $\mathcal{C}_{2}$-injetivos. mos, ainda, que temos um monomorfismo irredutível $P_{d} \longrightarrow P_{c}$, que não cinde. Portanto, $\mathcal{I}_{2}=\left\{P_{c}, S_{c}\right\}$.

Seja $\mathcal{C}_{3}=i n d \Lambda \backslash\left(\mathcal{I}_{0} \cup \mathcal{I}_{1} \cup \mathcal{I}_{2}\right)$.

$E$, por fim, $\mathcal{I}_{3}=\left\{P_{d}\right\}$.

Notemos que $\mathcal{I}_{0}, \mathcal{I}_{1}, \mathcal{I}_{2}, \mathcal{I}_{3}$ forma uma partição finita de ind $\Lambda$ e cada um deles contém apenas um número finito de elementos.

Exemplo 3.4.3. Considere a álgebra do Exemplo 2.2.4. A álgebra $\Lambda=\frac{k \Delta}{I}$, onde $\Delta$ é o quiver

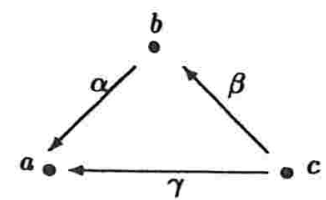

$$
e I=<\alpha \beta>
$$

O quiver de Auslander-Reiten $\Gamma_{\Lambda}$ é: 


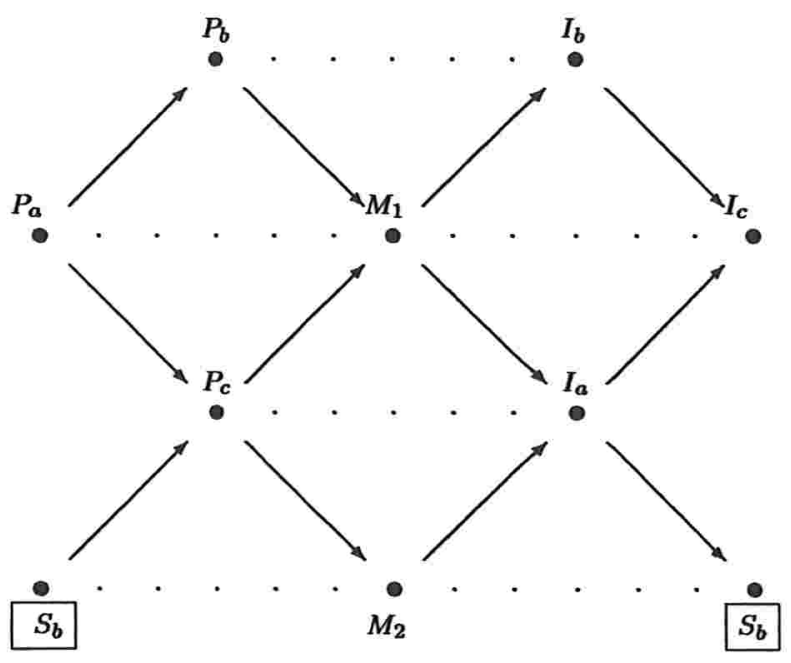

onde identificamos as duas cópias de $S_{b}$.

Realizando os cálculos concluímos que: $\mathcal{I}_{0}=\left\{I_{a}, I_{b}, I_{c}\right\}, \mathcal{I}_{1}=\left\{M_{1}, M_{2}\right\}, \mathcal{I}_{2}=\left\{P_{b}, P_{c}\right\}$ $e \mathcal{I}_{3}=\left\{P_{a}, S_{b}\right\}$.

Assim, $\mathcal{I}_{0}, \mathcal{I}_{1}, \mathcal{I}_{2}, \mathcal{I}_{3}$ forma uma partição pré-injetiva finita de ind $\Lambda$.

Lembramos que existe a noção dual de partição pré-injetiva, que é a partição pósprojetiva. A definição e os resultados dessa partição podem ser encontrados em [AS]. 


\section{Capítulo 4}

\section{Medida de Gabriel-Roiter para a álgebra de Kronecker}

Neste capítulo, faremos o estudo da medida de Gabriel-Roiter para todos os módulos da álgebra de Kronecker, que é uma álgebra de tipo representação infinito. Nessa álgebra os módulos apresentam uma estrutura bastante conhecida e suas medidas de Gabriel-Roiter estão bem estabelecidas.

Seja $k$ um corpo algebricamente fechado. Consideremos a álgebra $\Lambda=k \Delta$, onde $\Delta$ é o quiver

$$
a \stackrel{\alpha}{\stackrel{\alpha}{\longrightarrow}} \cdot b
$$

que é a conhecida álgebra de Kronecker.

Existem apenas três tipos de módulos indecomponíveis sobre a álgebra de Kronecker, conforme mostra seguinte teorema:

Teorema 1. Seja $\Lambda$ a álgebra de Kronecker. Os $\Lambda$-módulos indecomponíveis são dos seguintes tipos de representação:

(I) Para cada $n \geq 0$,

$Q_{n}: k^{n+1} \bullet \underset{g_{\beta, n}}{\stackrel{g_{\alpha, n}}{\longrightarrow}} \cdot k^{n} \quad$ onde $g_{\alpha, n}=\left[\begin{array}{ll}I d_{n} & 0\end{array}\right]$ e $g_{\beta, n}=\left[\begin{array}{ll}0 & I d_{n}\end{array}\right]$, sendo $I d_{n} a$ matriz identidade de ordem $n$. 


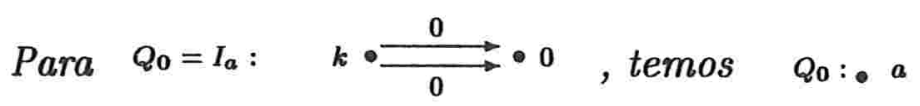

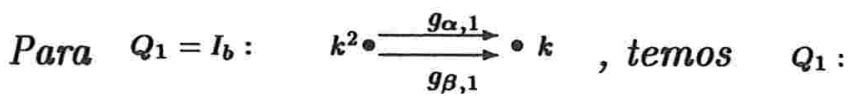

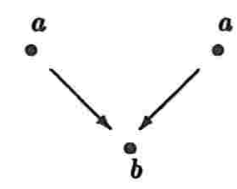

Para $n>1, \quad Q_{n}: k^{n+1} \cdot \underset{g_{\beta, n}}{\stackrel{g_{\alpha, n}}{\longrightarrow}} \cdot k^{n}$, temos

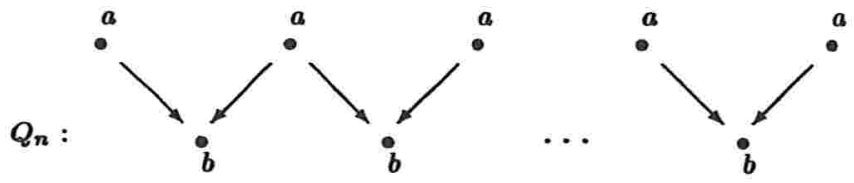

Notemos que na figura acima temos $n+1$ vértices a e $n$ vértices $b$.

(II) Para cada $n \geq 0$,

$P_{n}: k^{n_{0}} \underset{f_{\beta, n}}{\stackrel{f_{\alpha, n}}{\longrightarrow}} \cdot k^{n+1} \quad$ onde $f_{\alpha, n}=\left[\begin{array}{r}I d_{n} \\ 0\end{array}\right]$ e $f_{\beta, n}=\left[\begin{array}{r}0 \\ I d_{n}\end{array}\right]$, sendo $I d_{n}$ a matriz identidade de ordem $n$.

Para $\quad P_{0}=P_{b}: \quad 0 \stackrel{0}{\stackrel{0}{\longrightarrow}} \cdot k$, temos $P_{0}: \bullet b$

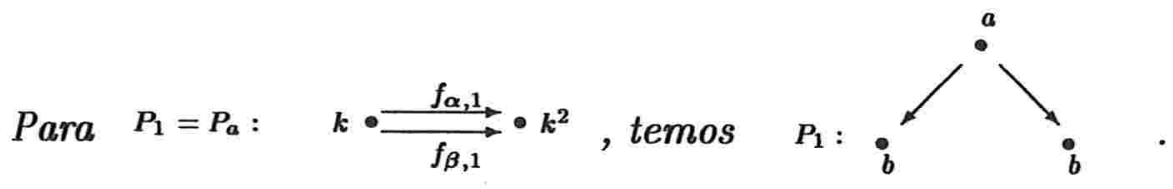

Para $n>1, \quad P_{n}: k^{n} \bullet \underset{f_{\beta, n}}{\stackrel{f_{\alpha, n}}{\longrightarrow}} \cdot k^{n+1} \quad$, temos

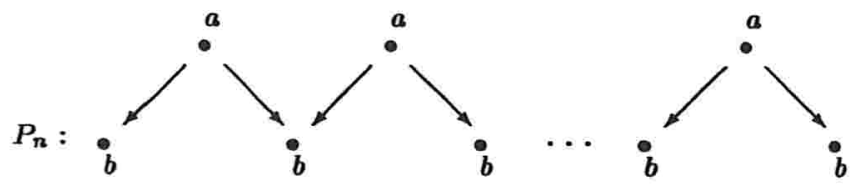

Notemos que, na figura acima temos $n$ vértices a e $n+1$ vértices $b$.

(III) Para cada $n \geq 0$ e $\lambda \in k$,

$R_{n}^{\lambda}: \quad k^{n} \bullet \underset{J_{n}(\lambda)}{\stackrel{I d_{n}}{\longrightarrow}} \cdot k^{n} \quad J_{n}(\lambda)$ é a matriz forma de Jordan.

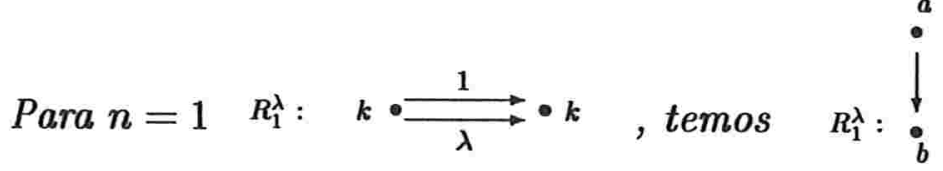


Para $n=2 \quad R_{2}^{\lambda}: \quad k^{2} \bullet \stackrel{\frac{I d_{2}}{J_{2}(\lambda)}}{\longrightarrow} k^{2}$, temos $R_{2}^{\lambda}:$

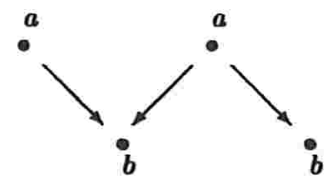

Para $n>1 \quad R_{n}^{\lambda}: \quad k^{n} \stackrel{\stackrel{I d_{n}}{\longrightarrow}}{\underset{J_{n}(\lambda)}{\longrightarrow}} \cdot k^{n}$, temos $R_{n}^{\lambda}$ :

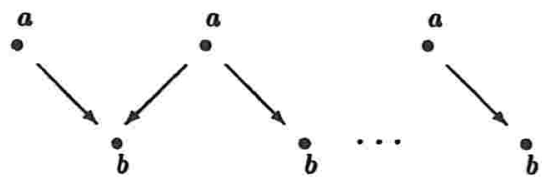

Notemos que, para $R_{n}^{\lambda}$, temos $n$ vértices tanto para a como para $b$.

Não faremos a demonstração do resultado acima por fugir de nossos objetivos aqui, porém, ela pode ser encontrada em [ARS].

Observação 1. Os módulos do tipo $(I)$ correspondem aos módulos pré-injetivos definidos na seção 3.4. Os módulos do tipo (II), aos duais dos pré-injetivos são módulos chamados pós-projetivos e os dos tipos (III), que não são nem pré-injetivos, nem pós-projetivos, são os chamados módulos regulares. Maiores detalhes a respeito da definição e propriedades desses módulos, podem ser encontradas em [AS].

Vejamos o quiver de Auslander-Reiten:

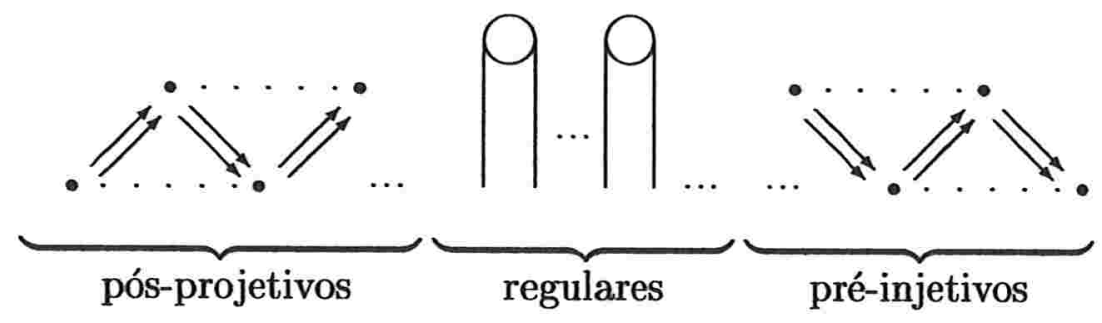

Calculemos agora a medida de Gabriel-Roiter para os $\Lambda$-módulos indecomponíveis da álgebra de Kronecker.

(i) módulos pré-injetivos

$$
\mu\left(Q_{0}\right)=\mu\left(I_{a}\right)=\mu\left(S_{a}\right)=\{1\}
$$


$\mu\left(Q_{1}\right)=\mu\left(I_{b}\right)=\{1,2,3\}$

$\mu\left(Q_{n}\right)=\{1,2, \ldots, 2 n, 2 n+1\}$.

Notemos que $\ell\left(Q_{n}\right)=2 n+1$.

(ii) módulos pós-projetivos.

$$
\begin{aligned}
& \mu\left(P_{0}\right)=\mu\left(P_{b}\right)=\mu\left(S_{b}\right)=\{1\} \\
& \mu\left(P_{1}\right)=\mu\left(P_{a}\right)=\{1,3\} \\
& \mu\left(P_{n}\right)=\{1,3, \ldots, 2 n+1\} .
\end{aligned}
$$

Notemos que $\ell\left(P_{n}\right)=2 n+1$.

(iii) módulos regulares

$$
\begin{aligned}
& \mu\left(R_{1}^{\lambda}\right)=\{1,2\} \\
& \mu\left(R_{n}^{\lambda}\right)=\{1,2, \ldots, 2 n\} .
\end{aligned}
$$

Notemos que $\ell\left(R_{n}^{\lambda}\right)=2 n$ e para cada $i=1,2, \ldots$, se $\lambda \neq \lambda^{\prime}$, então $R_{i}^{\lambda}$ e $R_{i}^{\lambda^{\prime}}$ são módulos indecomponíveis não isomorfos. Portanto, temos que existem uma infinidade de módulos cuja medida de Gabriel-Roiter é $\{1,2\}$; analogamente para as medidas $\{1,2, \ldots, 2 n\}$, com $n \geq 1$.

Para ordenar os módulos de Kronecker, usaremos a ordenação das medidas de GabrielRoiter para os módulos de Kronecker e aplicaremos a função $r$ da Proposição 2.2.12, que irá relacioná-las com os números racionais. Temos:

$$
\begin{aligned}
& r\left(\mu\left(P_{1}\right)\right)=r(\{1,3\})=\frac{1}{4} \\
& r\left(\mu\left(P_{2}\right)=r(\{1,3,5\})=\frac{5}{16}\right. \\
& r\left(\mu\left(P_{n}\right)\right)=r(\{1,3, \ldots, 2 n+1\})=\frac{1}{3}\left(1-\frac{1}{4^{n}}\right) \\
& r\left(\mu\left(Q_{1}\right)\right)=r(\{1,2,3\})=\frac{3}{4} \\
& r\left(\mu\left(Q_{2}\right)\right)=r(\{1,2,4,5\})=\frac{11}{16} \\
& r\left(\mu\left(Q_{n}\right)\right)=r(\{1,2,4, \ldots, 2 n, 2 n+1\})=\frac{2}{3}\left(1-\frac{1}{4^{n}}\right)+\frac{1}{4^{n}} \\
& r\left(\mu\left(R_{1}^{\lambda}\right)\right)=r(\{1,2\})=\frac{1}{2} \\
& r\left(\mu\left(R_{2}^{\lambda}\right)\right)=r(\{1,2,4\})=\frac{5}{8}
\end{aligned}
$$


$r\left(\mu\left(R_{n}^{\lambda}\right)\right)=r(\{1,2,4, \ldots, 2 n\})=\frac{2}{3}\left(1-\frac{1}{4^{n}}\right)$.

Notemos que $\mu\left(S_{a}\right)=\mu\left(S_{b}\right)=\{1\}$ e $r(\{1\})=0$ e ainda para $n \rightarrow \infty$ temos

$r\left(\mu\left(P_{n}\right)\right) \rightarrow \frac{1}{3}$, notemos que as medidas dos módulos pós-projetivos se aproximam pela esquerda de $\frac{1}{3}$.

$r\left(\mu\left(Q_{n}\right)\right) \rightarrow \frac{2}{3}$, notemos que as medidas dos módulos pré-injetivos se aproximam pela direita de $\frac{2}{3}$, pois as medidas dos módulos estão decrescendo.

$r\left(\mu\left(R_{n}^{\lambda}\right)\right) \rightarrow \frac{2}{3}$, notemos que as medidas dos módulos regulares se aproximam pela esquerda de $\frac{2}{3}$.

Segue que existem dois pontos de acumulação. Eles correspondem às medidas de Gabriel-Roiter dos módulos infinitamente gerados. Então temos a ordenação conforme indicado abaixo:

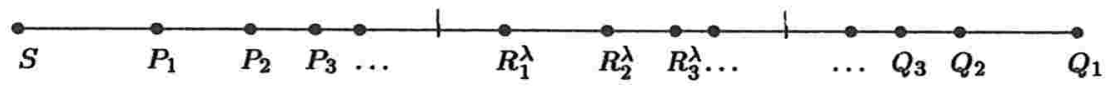

onde $S=\left\{S_{a}, S_{b}\right\}$. Observemos que os pontos de acumulação estão representados por uma linha vertical na reta. 


\section{Referências Bibliográficas}

[A] I. Assem, Algèbres et modules- cours et exercices, Masson, Le Presses de l'Université d'Ottawa, 1997, $330 \mathrm{p}$.

[AF] F. Anderson, K. Fuller, Rings and categories of modules, Springer GTM 13, Springer-Verlag, New York-Heidelberg-Berlin (1973).

[ARS] M. Auslander, I. Reiten, S. Smal $\varnothing$, Representation theory of artin algebras, Cambridge Studies in Advanced Mathematics 36, Cambridge Univ. Press, 1995.

[AS] M. Auslander, S. Smalø, Preprojective modules over artin algebras, J. Algebra 66, (1980), 61-122.

[Au] M. Auslander, Representation theory of artin algebras II, Comm. in Algebra, 2 (1974) $269-310$.

[Co] F. U. Coelho, Uma introdução à teoria de representações de álgebras (minicurso), Atas da XII Escola de Álgebra, Diamantina (1992), 60p.

[CLS] C. Cibils, F. Larrión, L. Salmerón. Métodos diagramáticos en teoría de representaciones, UNAM, México, 1982.

[DK] Y. A. Drozd, V. V. Kirichenko, Finite Dimensional Algebras. Springer-Verlag, Berlin, 1994.

[Ga] P. Gabriel, Indecomposable Representation II, Symposia Mathematica, Vol XI. Academic Press, London / New York, (1973), 81 - 104.

[Ja] J. P. Jans, On the indecomposable representations of algebras, Ann. Math.66 (1957) $418-429$.

[JM] A. R. Jones, H. A. Merklen, Representações de álgebras: métodos diagramáticos, 
Publicações do Instituto de Matemática e Estatística da Universidade de São Paulo, São Paulo, 1984.

[Po] F. C. Polcino Milies, Anéis e módulos, Publicações do Instituto de Matemática e Estatística da Universidade de São Paulo, São Paulo, 1972.

[Ri] C. M. Ringel, The Gabriel-Roiter Measure. Bull. Soc. Math. France, a aparecer.

[Ri2] C. M. Ringel, Foundation of the Representation Theory of Artin Algebras, Using the Gabriel-Roiter Measure. Proceeding of the 36th. Symposium Ring theory and Representation theory, Vol 2 (2004) 1-19.

[Ro] A. V. Roiter, Unboundedness of the dimension of the indecomposable representations of an algebra which has infinitely many indecomposable representations. Izv. Akad. Nauk SSSR. Ser. Mat. (1968), 1275-1282.

[Ya] K. Yamagata, On artinian rings of finite representation type. J. Algebra, 50 (1978) 276-283. 